ALEA, Lat. Am. J. Probab. Math. Stat. 16, 633-664 (2019)

\title{
Berry-Esséen bounds for parameter estimation of general Gaussian processes
}

\section{Soukaina Douissi, Khalifa Es-Sebaiy and Frederi G. Viens}

Faculty of Sciences Semlalia,

University Cadi Ayyad,

Boulevard Abdelkrim Al Khattabi,

Marrakech 40000, Morocco.

E-mail address: douissi.soukaina@gmail.com

URL: https://sites .google.com/view/soukaina-douissi

Department of Mathematics,

Faculty of Science,

Kuwait University, Kuwait.

E-mail address: khalifa.essebaiy@ku.edu.kw

URL: https://sites.google.com/view/khalifa--es-sebaiy

Department of Statistics and Probability,

Michigan State University

619 Red Cedar Rd East Lansing, MI 48824, USA.

E-mail address: viens@msu.edu

URL: https://www.stt.msu.edu/ viens/

\begin{abstract}
We study rates of convergence in central limit theorems for the partial sum of squares of general Gaussian sequences, using tools from analysis on Wiener space. No assumption of stationarity, asymptotically or otherwise, is made. The main theoretical tool is the so-called Optimal Fourth Moment Theorem (Nourdin and Peccati, 2015), which provides a sharp quantitative estimate of the total variation distance on Wiener chaos to the normal law. The only assumptions made on the sequence are the existence of an asymptotic variance, that a least-squarestype estimator for this variance parameter has a bias and a variance which can be controlled, and that the sequence's auto-correlation function, which may exhibit long memory, has a no-worse memory than that of fractional Brownian motion with Hurst parameter $H<3 / 4$. Our main result is explicit, exhibiting the tradeoff between bias, variance, and memory. We apply our result to study drift parameter estimation problems for subfractional Ornstein-Uhlenbeck and bifractional Ornstein-Uhlenbeck processes with fixed-time-step observations. These are processes which fail to be stationary or self-similar, but for which detailed calculations result in explicit formulas for the estimators' asymptotic normality.
\end{abstract}

Received by the editors February 12th, 2018; accepted April 15th, 2019.

2010 Mathematics Subject Classification. 60F05, 60G15, 60H05, 60H07, 62F12.

Key words and phrases. Central limit theorem, Berry-Esseen, Nourdin-Peccati analysis, parameter estimation, fractional Brownian motion, long memory. 


\section{Introduction}

This paper presents the estimation of the asymptotic variance, when it exists, of a general Gaussian sequence, and applies it to non-stationary stochastic processes based on fractional noises, which can have long memory. We choose to work with a simple method based on empirical second moments, which is akin to the discretization of a least-squares method for the corresponding processes using discrete observations with no in-fill assumption (fixed time step).

Our work responds to the recent preprint Es-Sebaiy and Viens $(2019+)$, which was the first instance in which this type of estimation was performed without in-fill assumptions on the data, but which insisted on keeping the data stationary or asymptotically so. That paper Es-Sebaiy and Viens $(2019+)$, available on arXiv, contains an extensive description of the literature on parameter estimation for Gaussian stochastic processes. We do not repeat that analysis here. Instead, we list a number of recent articles which deal with various versions of least-squarestype parameter estimation for fractional-noise-driven Ornstein-Uhlenbeck processes (fOU-type), since that class of processes is similar to the various examples we consider in this article: Hu and Nualart (2010), Azmoodeh and Morlanes (2015), Azmoodeh and Viitasaari (2015), El Onsy et al. (2017b), Hu and Song (2013), Brouste and Iacus (2013), Neuenkirch and Tindel (2014), Belfadli et al. (2011), El Machkouri et al. (2016) and El Onsy et al. (2017a). respectively. Those papers are described in the context of our motivations in Es-Sebaiy and Viens $(2019+)$. We now highlight the distinction between our paper and Es-Sebaiy and Viens $(2019+)$.

In that work, the assumption of stationarity of the data was weakened by asking for asymptotic stationarity, where the deviation from a stationary model converged to 0 exponentially fast. This strong assumption only allowed to work with specific examples which are close to stationary. In that article, Ornstein-Uhlenbeck models were covered, as long as the driving noise was stationary, as it would be for standard fractional Brownian motion. That paper was the first instance in which parameters for fOU-type processes could be estimated with asymptotic normality using only discrete data with fixed time step. However, this range of application in Es-Sebaiy and Viens $(2019+)$ extended to non-stationary processes which benefited from an exponential ergodicity property. One leaves this range of applicability when driving OU equations with noises which are not stationary to begin with. This is our primary motivation in this article: to develop a framework in which no assumption of stationarity is made at the level of driving noise processes. Instead, we investigate a set of arguably minimal assumptions on how to estimate an asymptotic variance, if it exists, for any discretely observed Gaussian process. In this article, we make no a-priori assumption about the speed of convergence of the data to a stationary model, only asking that convergence occurs.

This implies that the main result of the paper, our estimator's asymptotic normality, applies to some of the more exotic non-stationary covariance structures, for which the estimator properties cannot be handled by any classical or current method. Our main result isolates three terms in order to construct estimates which are fully quantitative. We show that the total variation distance between the CLT scaling of our discrete-observation least-squares estimator of the asymptotic variance and a normal law is bounded above by the sum of a bias term, a variance term, and a term which comes from an application of the optimal fourth moment theorem of Nourdin and Peccati (2015), and depends only on an upper bound on 
the covariance structure of the discrete observations. This allows us to formulate clear assumptions on what is needed to turn this estimate into a quantitative asymptotic normality theorem for the estimator, in total variation distance. The advantage of working in this fashion is that one has an immediate way of identifying possible mean-variance-correlation trade-offs, since the corresponding three terms are explicit and are simply added together as a measure of how fast the estimator converges to a normal. There are additional features to our method which are described in Section 3, such as our ability to separate our minimal convergence assumptions between what is needed for strong consistency and what is needed in addition to obtain asymptotic normality: see Hypotheses $(\mathcal{H} 1)$ thru $(\mathcal{H} 4)$ in Section 3, the bullet points that follow, and the statements of Theorems 3.3 and 3.5 in the same section.

When applying our general method from Section 3 to specific examples in Section 4, we consider the solutions of the OU equation driven by sub-fractional Brownian motion and by bi-fractional Brownian motion, both of which have somewhat non-trivial covariance structures with non-stationary increments, which are then inherited by the corresponding fOU-type processes whose drift parameter $\theta$ is of interest. These fOU-type processes have been well studied in the literature over the last decade, including efforts to understand their statistical inference. We refer only to El Machkouri et al. (2016) for information about these processes and additional references. We compute the asymptotic variances of these fOU-type processes, which are explicit functions $f(\theta)$, depending only on $\theta$ (and on the Hurst-type parameters which are assumed to be known), and are in fact power functions of $\theta$. The normal asymptotics for the estimators of each of these two $f(\theta)$ 's can be converted to similar results for $\theta$ itself; see Es-Sebaiy and Viens $(2019+)$ for instance for an analysis, which we do not explore further here. Part of being able to apply the strategy of Section 3 successfully in the examples of Section 4, relies on three things, the first two which cannot be avoided, the third which enables an efficient estimation of convergence speeds:

(1) being able to identify $f(\theta)$, preferably in an explicit way, thus giving access to $\theta$, but at least to show that the data stream's variance converges;

(a) we do this for both the sub-fractional and bi-fractional OU processes;

(b) the expressions for $f(\theta)$ are simple (see the formulas for $f_{H}(\theta)$ and $f_{H, K}(\theta)$ for each process respectively, in (4.3) and (4.8)) but proving convergence and evaluating the speed therein is slightly non-trivial;

(2) showing that the CLT-normalized estimator of $f(\theta)$ has an asymptotic variance $\sigma^{2}$; this does not need to be found explicitly, as long as one can prove that the variances converge;

(a) we succeed in this task for both the sub-fractional and bi-fractional OU processes, and are moreover able to compute $\sigma^{2}$ semi-explicitly, using a series representation based on the covariance function of discrete-time fractional Gaussian noise (fGn); see formulas (4.7) and (4.9) respectively;

(b) these expressions comes as something of a surprise, since a priori there was no reason to expect that the estimator's asymptotic variance could be expressed using the fGn's covariance rather than the non-stationary covariance structure of the increments of the sub- or bi-fractional processes; 
(3) computing speeds of convergence of both the estimator's bias and its asymptotic variance, as explicitly as possible;

(a) this is done for both the sub-fractional and bi-fractional OU processes, in a rather explicit fashion, and requires a certain amount of elbow grease, where most calculations are relegated to lemmas in the Appendix;

(b) we do not claim to have performed these estimations as efficiently as can possibly be done; however, we think that the power orders of these convergences are probably optimal because: (i) we have based the estimations on the optimal fourth moment theorem of Nourdin and Peccati Nourdin and Peccati (2015), (ii) we have confirmed a phenomenon observed in a simpler (stationary) context in Neufcourt and Viens (2016) (third moment theorem) by which the speed of convergence is given by that of an absolute third moment rather than by a fourth cumulant, and (iii) our final result for the sub- and bi-fractional processes identify a well-known threshold of a Hurst parameter not exceeding $3 / 4$ as the upper endpoint of the range where quadratic variations are asymptotically normal.

To finish this introduction, we note the general structure of this paper. The next section presents preliminaries regarding the analysis of Wiener space, and other convenient facts, which are needed in some technical parts of the paper. The reader interested in our results more than our proofs can safely skip this Section 2 upon a first reading. Section 3 presents the general framework of how to estimate the asymptotic variance of a Gaussian process observed in discrete time. Section 4 shows how this method can be implemented in two cases of discretely observed OU processes driven by two well-known non-stationary Gaussian processes with medium-to-long memory. The Appendix collects calculations useful in Section 4.

\section{Preliminaries, and elements of analysis on Wiener space}

This section provides essential facts from basic probability and the analysis on Wiener space and the Malliavin calculus. These facts and their corresponding notations underlie all the results of this paper, but most of our results and arguments can be understood independently of this section. The interested reader can find more details in Nualart (2006, Chapter 1) and Nourdin and Peccati (2012, Chapter 2).

2.1. A convenient lemma. The following result is a direct consequence of the BorelCantelli Lemma (the proof is elementary; see e.g. Kloeden and Neuenkirch, 2007, Lemma 2.1). It is convenient for establishing almost-sure convergences from $L^{p}$ convergences.

Lemma 2.1. Let $\gamma>0$. Let $\left(Z_{n}\right)_{n \in \mathbb{N}}$ be a sequence of random variables. If for every $p \geqslant 1$ there exists a constant $c_{p}>0$ such that for all $n \in \mathbb{N}$,

$$
\left\|Z_{n}\right\|_{L^{p}(\Omega)} \leqslant c_{p} \cdot n^{-\gamma}
$$

then for all $\varepsilon>0$ there exists a random variable $\eta_{\varepsilon}$ which is almost surely finite such that

$$
\left|Z_{n}\right| \leqslant \eta_{\varepsilon} \cdot n^{-\gamma+\varepsilon} \quad \text { almost surely }
$$


for all $n \in \mathbb{N}$. Moreover, $E\left|\eta_{\varepsilon}\right|^{p}<\infty$ for all $p \geqslant 1$.

A typical application is when a sequence in a finite sum of Wiener chaoses converges in the mean square. By the equivalence of all $L^{p}$ norms in Wiener chaos, which is a consequence of the hypercontractivity of the Ornstein-Uhlenbeck semigroup on Wiener space (see Nourdin and Peccati, 2012, Section 2.8, and Section 2.3 below), the lemma's assumption is then automatically satisfied if the speed of convergence in the mean square is of the same power form.

The random variable $\eta_{\varepsilon}$ can typically be chosen more explicitly than what the lemma guarantees. For instance, it is well known that if $Z$ is Gaussian, then $\eta_{\varepsilon}$ can be chosen as a gauge function of $Z$, and is therefore sub-Gaussian (tails no larger than Gaussian; see Fernique, 1975). A similar property holds for processes in a finite sum of Wiener chaoses, as can be inferred from Viens and Vizcarra (2007). We will not pursue these refinements, since the above lemma will be sufficient for our purposes herein.

2.2. The Young integral. Fix $T>0$. Let $f, g:[0, T] \longrightarrow \mathbb{R}$ be Hölder continuous functions of orders $\alpha \in(0,1)$ and $\beta \in(0,1)$ respectively with $\alpha+\beta>1$. Young (1936) proved that the Riemann-Stieltjes integrals (so-called Young integrals) $\int_{0}^{\cdot} f_{u} d g_{u}$ and $\int_{0}^{\cdot} g_{u} d f_{u}$ exist as limits of the usual discretization. Moreover, for all $t \in[0, T]$, integration by parts (product rule) holds:

$$
f_{t} g_{t}=f_{0} g_{0}+\int_{0}^{t} g_{u} d f_{u}+\int_{0}^{t} f_{u} d g_{u}
$$

This integral is convenient to define stochastic integrals in a pathwise sense with respect to processes $f$ which are smoother than Brownian motion, in the sense that they are almost surely $\alpha$-Hölder continuous for some $\alpha>1 / 2$. A typical example of such a process is the fractional Brownian motion with Hurst parameter $H>1 / 2$. In that case, any $\alpha$ can be chosen in $(1 / 2, H)$, and $g$ can be any process with the same regularity as the $\mathrm{fBm}$, and thus with $\beta=\alpha$; this enables a stochastic calculus immediately, in which no "Itô"-type correction terms occur, owing to (2.1). For instance, for any Lipshitz non-random function $g$, this integration-by-parts formula holds for any a.s. Hölder-continuous Gaussian process $f$, since then $\alpha>0$ and $\beta=1$. In particular, the first integral in (2.1) coincides with the Wiener integral, the second is an ordinary Riemann-Stieltjes integral, and no Itô correction occurs since one of the processes is of finite variation.

2.3. Elements of Analysis on Wiener space. With $(\Omega, \mathcal{F}, \mathbf{P})$ denoting the Wiener space of a standard Wiener process $W$, for a deterministic function $h \in L^{2}\left(\mathbf{R}_{+}\right)=$: $\mathcal{H}$, the Wiener integral $\int_{\mathbf{R}_{+}} h(s) d W(s)$ is also denoted by $W(h)$. The inner product $\int_{\mathbf{R}_{+}} f(s) g(s) d s$ will be denoted by $\langle f, g\rangle_{\mathcal{H}}$.

- The Wiener chaos expansion. For every $q \geqslant 1, \mathcal{H}_{q}$ denotes the $q$ th Wiener chaos of $W$, defined as the closed linear subspace of $L^{2}(\Omega)$ generated by the random variables $\left\{H_{q}(W(h)), h \in \mathcal{H},\|h\|_{\mathcal{H}}=1\right\}$ where $H_{q}$ is the $q$ th Hermite polynomial. Wiener chaos of different orders are orthogonal in $L^{2}(\Omega)$. The so-called Wiener chaos expansion is the fact that any $X \in$ 
$L^{2}(\Omega)$ can be written as

$$
X=\mathbf{E} X+\sum_{q=0}^{\infty} X_{q}
$$

for some $X_{q} \in \mathcal{H}_{q}$ for every $q \geqslant 1$. This is summarized in the directorthogonal-sum notation $L^{2}(\Omega)=\oplus_{q=0}^{\infty} \mathcal{H}_{q}$. Here $\mathcal{H}_{0}$ denotes the constants.

- Relation with Hermite polynomials. Multiple Wiener integrals. The mapping $I_{q}\left(h^{\otimes q}\right):=q ! H_{q}(W(h))$ is a linear isometry between the symmetric tensor product $\mathcal{H}^{\odot q}$ (equipped with the modified norm $\|\cdot\|_{\mathcal{H} \odot q}=$ $\left.\sqrt{q !}\|\cdot\|_{\mathcal{H} \otimes q}\right)$ and $\mathcal{H}_{q}$. To relate this to standard stochastic calculus, one first notes that $I_{q}\left(h^{\otimes q}\right)$ can be interpreted as the multiple Wiener integral of $h^{\otimes q}$ w.r.t. $W$. By this we mean that the Riemann-Stieltjes approximation of such an integral converges in $L^{2}(\Omega)$ to $I_{q}\left(h^{\otimes q}\right)$. This is an elementary fact from analysis on Wiener space, which can also be proved using standard stochastic calculus for square-integrable martingales, because the multiple integral interpretation of $I_{q}\left(h^{\otimes q}\right)$ as a Riemann-Stieltjes integral over $\left(\mathbf{R}_{+}\right)^{q}$ can be further shown to coincide with $q$ ! times the iterated Itô integral over the first simplex in $\left(\mathbf{R}_{+}\right)^{q}$.

More generally, for $X$ and its Wiener chaos expansion (2.2) above, each term $X_{q}$ can be interpreted as a multiple Wiener integral $I_{q}\left(f_{q}\right)$ for some $f_{q} \in \mathcal{H}^{\odot q}$.

- The product formula - Isometry property. For every $f, g \in \mathcal{H}^{\odot q}$ the following extended isometry property holds

$$
E\left(I_{q}(f) I_{q}(g)\right)=q !\langle f, g\rangle_{\mathcal{H} \otimes q} .
$$

Similarly as for $I_{q}\left(h^{\otimes q}\right)$, this formula is established using basic analysis on Wiener space, but it can also be proved using standard stochastic calculus, owing to the coincidence of $I_{q}(f)$ and $I_{q}(g)$ with iterated Itô integrals. To do so, one uses Itô's version of integration by parts, in which iterated calculations show coincidence of the expectation of the bounded variation term with the right-hand side above. What is typically referred to as the Product Formula on Wiener space is the version of the above formula before taking expectations (see Nourdin and Peccati, 2012, Section 2.7.3). In our work, beyond the zero-order term in that formula, which coincides with the expectation above, we will only need to know the full formula for $q=1$, which is $I_{1}(f) I_{1}(g)=2^{-1} I_{2}(f \otimes g+g \otimes f)+\langle f, g\rangle_{\mathcal{H}}$.

- Hypercontractivity in Wiener chaos. For $h \in \mathcal{H}^{\otimes q}$, the multiple Wiener integrals $I_{q}(h)$, which exhaust the set $\mathcal{H}_{q}$, satisfy a hypercontractivity property (equivalence in $\mathcal{H}_{q}$ of all $L^{p}$ norms for all $p \geqslant 2$ ), which implies that for any $F \in \oplus_{l=1}^{q} \mathcal{H}_{l}$ (i.e. in a fixed sum of Wiener chaoses), we have

$$
\left(E\left[|F|^{p}\right]\right)^{1 / p} \leqslant c_{p, q}\left(E\left[|F|^{2}\right]\right)^{1 / 2} \text { for any } p \geqslant 2 .
$$

It should be noted that the constants $c_{p, q}$ above are known with some precision when $F$ is a single chaos term: indeed, by Corollary 2.8.14 in Nourdin and Peccati (2012), $c_{p, q}=(p-1)^{q / 2}$.

- Malliavin derivative. The Malliavin derivative operator $D$ on Wiener space is not needed explicitly in the paper. However, because of the fundamental role $D$ plays in evaluating distances between random variables, 
it is helpful to introduce it, to justify the estimates (2.6) and (2.7) below. For any function $\Phi \in C^{1}(\mathbf{R})$ with bounded derivative, and any $h \in \mathcal{H}$, the Malliavin derivative of the random variable $X:=\Phi(W(h))$ is defined to be consistent with the following chain rule:

$$
D X: X \mapsto D_{r} X:=\Phi^{\prime}(W(h)) h(r) \in L^{2}\left(\Omega \times \mathbf{R}_{+}\right) .
$$

A similar chain rule holds for multivariate $\Phi$. One then extends $D$ to the so-called Gross-Sobolev subset $\mathbf{D}^{1,2} \varsubsetneqq L^{2}(\Omega)$ by closing $D$ inside $L^{2}(\Omega)$ under the norm defined by

$$
\|X\|_{1,2}^{2}=\mathbf{E}\left[X^{2}\right]+\mathbf{E}\left[\int_{\mathbf{R}_{+}}\left|D_{r} X\right|^{2} d r\right] .
$$

All Wiener chaos random variable are in the domain $\mathbf{D}^{1,2}$ of $D$. In fact this domain can be expressed explicitly for any $X$ as in (2.2): $X \in \mathbf{D}^{1,2}$ if and only if $\sum_{q} q q !\left\|f_{q}\right\|_{\mathcal{H} \otimes q}^{2}<\infty$.

- Generator $L$ of the Ornstein-Uhlenbeck semigroup. The linear operator $L$ is defined as being diagonal under the Wiener chaos expansion of $L^{2}(\Omega): \mathcal{H}_{q}$ is the eigenspace of $L$ with eigenvalue $-q$, i.e. for any $X \in \mathcal{H}_{q}$, $L X=-q X$. We have $\operatorname{Ker}(L)=\mathcal{H}_{0}$, the constants. The operator $-L^{-1}$ is the negative pseudo-inverse of $L$, so that for any $X \in \mathcal{H}_{q},-L^{-1} X=q^{-1} X$. Since the variables we will be dealing with in this article are finite sums of elements of $\mathcal{H}_{q}$, the operator $-L^{-1}$ is easy to manipulate thereon. The use of $L$ is crucial when evaluating the total variation distance between the laws of random variables in Wiener chaos, as we will see shortly.

- Distances between random variables. Recall that, if $X, Y$ are two real-valued random variables, then the total variation distance between the law of $X$ and the law of $Y$ is given by

$$
d_{T V}(X, Y)=\sup _{A \in \mathcal{B}(\mathbb{R})}|P[X \in A]-P[Y \in A]|
$$

where the supremum is over all Borel sets. Also, let us recall that, for $Y=$ $\mathcal{N}(0,1)$ and $X$ is an integrable random variable, another characterization of the total variation distance (see Nualart and Zhou, 2018, page 10, Nourdin and Peccati, 2012, Theorem 3.3.1) is given by

$$
d_{T V}(X, \mathcal{N}(0,1))=\sup _{f \in \mathcal{C}^{1}(\mathbb{R}),\|f\|_{\infty} \leq \sqrt{\pi / 2},\left\|f^{\prime}\right\|_{\infty} \leq 2}\left|E\left(f^{\prime}(X)-X f(X)\right)\right| .
$$

- Malliavin operators and distances between laws on Wiener space. There are two key estimates linking total variation distance and the Malliavin calculus, which were both obtained by Nourdin and Peccati. The first one is an observation relating an integration-by-parts formula on Wiener space with a classical result of Ch. Stein. The second is a quantitatively sharp version of the famous 4th moment theorem of Nualart and Peccati. Let $N$ denote the standard normal law.

- The observation of Nourdin and Peccati. Let $X \in \mathbf{D}^{1,2}$ with $\mathbf{E}[X]=0$ and $\operatorname{Var}[X]=1$. Then (see Nourdin and Peccati, 2015, Proposition 2.4, or Nourdin and Peccati, 2012, Theorem 5.1.3), for $f \in C_{b}^{1}(\mathbf{R})$,

$$
E[X f(X)]=E\left[f^{\prime}(X)\left\langle D X,-D L^{-1} X\right\rangle_{\mathcal{H}}\right]
$$


and by combining this with properties of solutions of Stein's equations, one gets

$$
d_{T V}(X, N) \leqslant 2 E\left|1-\left\langle D X,-D L^{-1} X\right\rangle_{\mathcal{H}}\right| .
$$

One notes in particular that when $X \in \mathcal{H}_{q}$, since $-L^{-1} X=q^{-1} X$, we obtain conveniently

$$
d_{T V}(X, N) \leqslant 2 E\left|1-q^{-1}\|D X\|_{\mathcal{H}}^{2}\right| .
$$

It is this last observation which leads to a quantitative version of the fourth moment theorem of Nualart and Peccati, which entails using Jensen's inequality to note that the right-hand side of (2.5) is bounded above by the variance of $\left\langle D X,-D L^{-1} X\right\rangle_{\mathcal{H}}$, and then relating that variance in the case of Wiener chaos with the 4th cumulant of $X$. However, this strategy was superseded by the following, which has roots in Biermé et al. (2012).

- The optimal fourth moment theorem. For each integer $n$, let $X_{n} \in \mathcal{H}_{q}$. Assume $\operatorname{Var}\left[X_{n}\right]=1$ and $\left(X_{n}\right)_{n}$ converges in distribution to a normal law. It is known (original proof in Nualart and Peccati, 2005, known as the fourth moment theorem) that this convergence is equivalent to $\lim _{n} \mathbf{E}\left[X_{n}^{4}\right]=3$. The following optimal estimate for $d_{T V}(X, N)$, known as the optimal fourth moment theorem, was proved in Nourdin and Peccati (2015): with the sequence $X$ as above, assuming convergence, there exist two constants $c, C>0$ depending only on $X$ but not on $n$, such that

$$
c \max \left\{\mathbf{E}\left[X_{n}^{4}\right]-3,\left|\mathbf{E}\left[X_{n}^{3}\right]\right|\right\} \leqslant d_{T V}\left(X_{n}, N\right) \leqslant C \max \left\{\mathbf{E}\left[X_{n}^{4}\right]-3,\left|\mathbf{E}\left[X_{n}^{3}\right]\right|\right\} .
$$

Given its importance, the centered fourth moment, also known as the fourth cumulant, of a standardized random variable, warrants the following special notation:

$$
\kappa_{4}(X):=\mathbf{E}\left[X^{4}\right]-3 .
$$

Let us also recall that if $E\left[F_{n}\right]=0$, we denote $\kappa_{3}\left(F_{n}\right)=E\left[F_{n}^{3}\right]$ its third cumulant. Throughout the paper we use the notation $N \sim \mathcal{N}(0,1)$. We also use the notation $C$ for any positive real constant, independently of its value which may change from line to line when this does not lead to ambiguity.

\section{General Context}

Let $G=\left\{G_{t}, t \geqslant 0\right\}$ be an underlying Gaussian process, and let $X=\left\{X_{n}\right\}_{n \geqslant 0}$ be a mean-zero Gaussian sequence measurable with respect to $G$. This means that $X$ is a sequence of random variables which can be represented as $X_{t}=I_{1}\left(h_{t}\right)$ for every $t \geqslant 0$, i.e. $X_{t}$ is a Wiener integral with respect to $G$, where $h_{t} \in \mathcal{H}$ with $\mathcal{H}$ the Hilbert space associated to $G$. In particular, we do not assume that $X$ is a stationary process. Define

$$
A_{n}(X):=\frac{1}{n} \sum_{i=1}^{n} E\left[X_{i}^{2}\right] \quad \text { and } \quad V_{n}(X):=\frac{1}{\sqrt{n}} \sum_{i=1}^{n}\left(X_{i}^{2}-E\left[X_{i}^{2}\right]\right) .
$$

As we explained in the introduction, the goal is to estimate the asymptotic variance $f$ of the sequence $X$, if it exists. Assuming it exists is the first of the following 
four assumptions, i.e. $(\mathcal{H} 1)$. The other three give us various levels of additional regularity for the law of $X$ to measure the speed of convergence of a quadraticvariations-based estimator for $f$ :

$(\mathcal{H} 1) E\left[X_{n}^{2}\right] \longrightarrow f$ as $n \longrightarrow \infty$.

$(\mathcal{H} 2) v_{n}(X):=E\left[V_{n}(X)^{2}\right] \longrightarrow \sigma^{2}>0$, as $n \longrightarrow \infty$.

$(\mathcal{H} 3)\left|E\left[X_{t} X_{s}\right]\right| \leqslant C \rho(|t-s|)$ where $\rho: \mathbb{R} \rightarrow \mathbb{R}$ is a symmetric positive function such that $\rho(0)=1$, and $C$ is a positive constant.

(H4) $\sqrt{n}\left|A_{n}(X)-f\right| \longrightarrow 0$, as $n \longrightarrow \infty$.

We consider the following estimator of $f$

$$
\hat{f}_{n}(X)=\frac{1}{n} \sum_{i=1}^{n} X_{i}^{2} .
$$

We refer to El Onsy et al. (2017b) and Es-Sebaiy and Viens $(2019+)$ for the construction of this estimator (3.1) for some classes of processes and sequences, i.e. how it can be interpreted as a discretization of a least squares estimator of $f$. The next two subsections study strong consistency and speed of convergence in the asymptotic normality of $\hat{f}_{n}(X)$. We make some comments on the roles and motivations behind the four assumptions above.

- As we said, Assumption $(\mathcal{H} 1)$ states that the asymptotic variance $f$ of the sequence $X$ exists.

- Assumption $(\mathcal{H} 2)$ helps establish strong consistency of $\hat{f}_{n}(X)$ via the BorelCantelli lemma and hypercontractivity of Wiener chaos. It states that a proper standardization of $\hat{f}_{n}(X)$ has an asymptotic variance; it is also used to help establish a quantitative upper bound on the total variation distance between a standardized version of $\hat{f}_{n}(X)$ and the standard normal law.

- Assumption $(\mathcal{H} 3)$ formalizes the idea that while the sequence $X$ is not stationary, it may have a covariance structure which is bounded above by one which may be comparable to a stationary one. This assumption itself is largely used as a matter of convenience, since formally by Schwartz's inequality it can always be assumed to hold for the trivial case $\rho \equiv 1$. However, by making further quantitative estimates on the rate of decay of $\rho$ to 0 , we find convenient explicit upper bound expressions for the total variation distance of between a standardization of $\hat{f}_{n}(X)$ and the normal law. The corresponding results are in Theorem 3.3.

- In particular, Assumption $(\mathcal{H} 4)$ quantifies the speed of convergence of the mean of $\hat{f}_{n}(X)$ towards $f$; combined with Assumption $(\mathcal{H} 2)$ which determines the speed of convergence of the variance of $\hat{f}_{n}(X)$, and using estimates on Wiener space, one can arrive at a fully quantified upper bound on the total variation distance between $\sqrt{n}\left(\hat{f}_{n}(X)-f\right)$ and the law $\mathcal{N}\left(0, \sigma^{2}\right)$. The corresponding results are in Theorem 3.5.

- The last point above is significant because Theorem 3.5 does not rely on using $v_{n}$, which is not observed, to standardize $\hat{f}_{n}(X)$. This theorem also decomposes the distance to the limiting normal law into the sum of three explicit terms: one to account for the bias of $\hat{f}_{n}(X)$ (from Assumption $(\mathcal{H} 4)$ ), one to account for the speed of convergence to the asymptotic variance (from Assumption $(\mathcal{H} 2)$ ), and one from the analysis on Wiener space 
which uses the speed of decay of the correlations of $X$ (from Assumption $(\mathcal{H} 3))$.

3.1. Strong consistency. The following theorem provides sufficient assumptions to obtain the estimator $\hat{f}_{n}(X)$ 's strong consistency, i.e. almost sure convergence to $f$.

Theorem 3.1. Assume that $(\mathcal{H} 1)$ and $(\mathcal{H} 2)$ hold. Then

$$
\hat{f}_{n}(X) \longrightarrow f
$$

almost surely as $n \longrightarrow \infty$.

Proof: It is clear that

$$
\hat{f}_{n}(X)=\frac{V_{n}(X)}{\sqrt{n}}+A_{n}(X) .
$$

The hypothesis $(\mathcal{H} 1)$ and the convergence of Cesàro means imply that, as $n \longrightarrow \infty$

$$
A_{n}(X) \longrightarrow f \text {. }
$$

In order to prove (3.2) it remains to check that, as $n \longrightarrow \infty, \frac{V_{n}(X)}{\sqrt{n}} \longrightarrow 0$ almost surely. According to $(\mathcal{H} 2)$, there exists $C>0$ such that for all $n>1$

$$
\left(E\left[\left|\frac{V_{n}(X)}{\sqrt{n}}\right|^{2}\right]\right)^{1 / 2} \leqslant \frac{C}{\sqrt{n}} .
$$

Now, using the hypercontractivity property (2.3) and Lemma 2.1 the result is obtained.

3.2. Asymptotic normality. In this section we study the asymptotic normality of $\hat{f}_{n}(X)$. By the product formula, we can write

$$
V_{n}(X)=\frac{1}{\sqrt{n}} \sum_{k=1}^{n}\left(X_{k}^{2}-E\left[X_{k}^{2}\right]\right)=\frac{1}{\sqrt{n}} \sum_{k=1}^{n} I_{2}\left(f_{k}^{\otimes 2}\right) .
$$

Set

$$
v_{n}(X):=E\left[V_{n}(X)^{2}\right]=\frac{2}{n} \sum_{j, k=1}^{n}\left(E\left[X_{j} X_{k}\right]\right)^{2},
$$

where the second equality is from elementary covariance calculation, and

$$
F_{n}(X):=\frac{V_{n}(X)}{\sqrt{v_{n}(X)}}=I_{2}\left(g_{n}\right)
$$

where

$$
g_{n}:=\left(n v_{n}(X)\right)^{-1 / 2} \sum_{k=1}^{n} f_{k}^{\otimes 2} .
$$

Let us estimate the third cumulant of $F_{n}(X)$. We have for every $n \geqslant 1$, by the observation of Nourdin and Peccati (or using calculus on Wiener chaos), we have

$$
\kappa_{3}\left(F_{n}(X)\right)=2 E\left[F_{n}(X) \Gamma_{1}\left(F_{n}(X)\right)\right],
$$


where $\Gamma_{1}\left(F_{n}(X)\right)=2 I_{2}\left(g_{n} \widetilde{\otimes}_{1} g_{n}\right)+2\left\|g_{n}\right\|_{\mathcal{H} \otimes 2}^{2}$. Assume that $(\mathcal{H} 3)$ holds. We then have

$$
\begin{aligned}
\kappa_{3}\left(F_{n}(X)\right) & =8\left\langle g_{n}, g_{n} \widetilde{\otimes}_{1} g_{n}\right\rangle_{\mathcal{H} \otimes 2}^{2} \\
& =8\left\langle g_{n}, g_{n} \otimes_{1} g_{n}\right\rangle_{\mathcal{H}^{\otimes 2}}^{2} \\
& =\frac{8}{\left(n v_{n}(X)\right)^{3 / 2}} \sum_{i, j, k=1}^{n}\left\langle f_{i}, f_{k}\right\rangle_{\mathcal{H}}\left\langle f_{i}, f_{j}\right\rangle_{\mathcal{H}}\left\langle f_{k}, f_{j}\right\rangle_{\mathcal{H}} \\
& =\frac{8}{\left(n v_{n}(X)\right)^{3 / 2}} \sum_{i, j, k=1}^{n} E\left[X_{i} X_{k}\right] E\left[X_{i} X_{j}\right] E\left[X_{j} X_{k}\right] .
\end{aligned}
$$

Therefore,

$$
\begin{aligned}
\left|\kappa_{3}\left(F_{n}(X)\right)\right| & \unlhd \frac{1}{\left(n v_{n}(X)\right)^{3 / 2}} \sum_{i, j, k=1}^{n}|\rho(i-k) \rho(i-j) \rho(j-k)| \\
& \unlhd \frac{1}{v_{n}(X)^{3 / 2} \sqrt{n}}\left(\sum_{|k|<n}|\rho(k)|^{3 / 2}\right)^{2} .
\end{aligned}
$$

The last equality follows from the same argument as for the proof of Biermé et al. (2012, Proposition 6.3). The symbol $\unlhd$ means we omit multiplicative universal constants.

Now for the fourth cumulant, similarly, we have

$$
\begin{aligned}
\kappa_{4}\left(F_{n}(X)\right) & =\frac{1}{v_{n}(X)^{2} n^{2}} \sum_{k, i, j, l=1}^{n} E\left[X_{k} X_{i}\right] E\left[X_{i} X_{j}\right] E\left[X_{j} X_{l}\right] E\left[X_{l} X_{k}\right] \\
& \unlhd \frac{1}{v_{n}(X)^{2} n^{2}} \sum_{k, i, j, l=1}^{n}|\rho(k-l) \rho(i-j) \rho(k-i) \rho(l-j)| \\
& \unlhd \frac{1}{v_{n}(X)^{2} n}\left(\sum_{|k|<n}|\rho(k)|^{\frac{4}{3}}\right)^{3}
\end{aligned}
$$

where again the last inequality comes from a similar argument as for the proof of Biermé et al. (2012, Proposition 6.4).

Remark 3.2. In Biermé et al. (2012, Proposition 6.3, Proposition 6.4) the sequence $\left\{X_{n}\right\}_{n \geqslant 0}$ is a centered stationary Gaussian sequence. In our case, it is not necessarily stationary. Our Hypothesis $(\mathcal{H} 3)$ is sufficient to avoid the assumption of stationarity.

Also note that there is no need to take the absolute value of $\kappa_{4}$ because the fourth cumulant of a variable in a fixed chaos $\left(F_{n}\right.$ is in the 2 nd chaos) is known to have a positive 4th cumulant.

Theorem 3.3. Let $N \sim \mathcal{N}(0,1)$ and assume that the hypothesis $(\mathcal{H} 3)$ holds. Then there exists a constant $C>0$ (which depends on the random sequence $F_{n}(X)$ but 
not on $n$ ) such that for all $n>1$,

$$
\begin{aligned}
& d_{T V}\left(F_{n}(X), N\right) \\
& \leqslant C \max \left\{\frac{1}{v_{n}(X)^{3 / 2} \sqrt{n}}\left(\sum_{|k|<n}|\rho(k)|^{3 / 2}\right)^{2}, \frac{1}{v_{n}(X)^{2} n}\left(\sum_{|k|<n}|\rho(k)|^{\frac{4}{3}}\right)^{3}\right\} .
\end{aligned}
$$

In particular, if for some $\beta \geqslant 1 / 2$, we have $\rho(t)=O\left(|t|^{-\beta}\right)$ for large $|t|$, then the following bound holds for all $n>1$

$$
d_{T V}\left(F_{n}(X), N\right) \leqslant \frac{C}{v_{n}(X)^{2} \wedge v_{n}(X)^{3 / 2}} \begin{cases}1 & \text { if } \beta=\frac{1}{2} \\ n^{\frac{3}{2}-3 \beta} & \text { if } \beta \in\left(\frac{1}{2}, \frac{2}{3}\right) \\ n^{-\frac{1}{2}} \log (n)^{2} & \text { if } \beta=\frac{2}{3} \\ n^{-\frac{1}{2}} & \text { if } \beta>\frac{2}{3} .\end{cases}
$$

Proof: The estimate (3.5) is a direct consequence of the optimal estimate in (2.7), and the estimates (3.3) and (3.4) of the absolute third and fourth cumulants we just computed. For (3.6), since

$$
\sum_{|k|<n}|\rho(k)|^{3 / 2} \leqslant C \begin{cases}n^{1 / 4} & \text { if } \beta=\frac{1}{2} \\ n^{1-\frac{3}{2} \beta} & \text { if } \beta \in\left(\frac{1}{2}, \frac{2}{3}\right) \\ \log n & \text { if } \beta=\frac{2}{3} \\ 1 & \text { if } \beta>\frac{2}{3}\end{cases}
$$

and

$$
\sum_{|k|<n}|\rho(k)|^{4 / 3} \leqslant C \begin{cases}n^{1 / 3} & \text { if } \beta=\frac{1}{2} \\ n^{\frac{-4}{3} \beta+1} & \text { if } \beta \in\left(\frac{1}{2}, \frac{3}{4}\right) \\ \log n & \text { if } \beta=\frac{3}{4} \\ 1 & \text { if } \beta>\frac{3}{4}\end{cases}
$$

the desired result is obtained.

Remark 3.4. The phenomenon observed in the proof of the previous theorem, by which the estimate of the third cumulant dominates that of the fourth cumulant, was observed originally in Biermé et al. (2012, Proposition 6.3, Proposition 6.4) for stationary sequences, and shown in Neufcourt and Viens (2016) to be completely general in the stationary case (i.e. not related to the power scale). Here we see that stationarity is not required. This begs the question of whether the phenomenon generalizes to other sequences in the second chaos, for instance without using hypothesis $(\mathcal{H} 3)$. We do not investigate this question here, since it falls well outside the scope of our motivating topic of parameter estimation. 
Theorem 3.5. Assume that the hypothesis $(\mathcal{H} 3)$ holds. If for some $\frac{1}{2} \leqslant \beta$, we have $\rho(t)=O\left(|t|^{-\beta}\right)$ for large $|t|$, then there exists a constant $C>0$ (which depends on $X$ but not on $n)$ such that for all $n>1$,

$$
\begin{aligned}
d_{T V}\left(\sqrt{\frac{n}{v_{n}}}\left(\hat{f}_{n}(X)-f\right), N\right) \leqslant & \sqrt{\frac{\pi n}{2 v_{n}}}\left|A_{n}(X)-f\right| \\
& +\frac{C}{v_{n}^{2} \wedge v_{n}^{3 / 2}} \begin{cases}1 & \text { if } \beta=\frac{1}{2} \\
n^{\frac{3}{2}-3 \beta} & \text { if } \beta \in\left(\frac{1}{2}, \frac{2}{3}\right) \\
n^{-\frac{1}{2}} \log (n)^{2} & \text { if } \beta=\frac{2}{3} \\
n^{-\frac{1}{2}} & \text { if } \beta>\frac{2}{3} .\end{cases}
\end{aligned}
$$

In addition if $(\mathcal{H} 2)$ holds, we have

$$
\begin{aligned}
& d_{T V}\left(\frac{\sqrt{n}}{\sigma}\left(\hat{f}_{n}(X)-f\right), N\right) \leqslant C\left(\sqrt{n}\left|A_{n}(X)-f\right|+\left|v_{n}-\sigma^{2}\right|\right) \\
&+C \begin{cases}n^{\frac{3}{2}-3 \beta} & \text { if } \beta \in\left(\frac{1}{2}, \frac{2}{3}\right) \\
n^{-\frac{1}{2}} \log (n)^{2} & \text { if } \beta=\frac{2}{3} \\
n^{-\frac{1}{2}} & \text { if } \beta>\frac{2}{3} .\end{cases}
\end{aligned}
$$

In particular, if the assumptions $(\mathcal{H} 2)-(\mathcal{H} 4)$ hold and for some $\beta>1 / 2$, we have $\rho(t)=O\left(|t|^{-\beta}\right)$ for large $|t|$, then, as $n \rightarrow \infty$

$$
\sqrt{n}\left(\hat{f}_{n}(X)-f\right) \stackrel{\text { law }}{\longrightarrow} \mathcal{N}\left(0, \sigma^{2}\right) .
$$

Proof: Theorem 3.5 is a direct consequence of Theorem 3.3, Lemma 5.1, and the fact that

$$
\sqrt{\frac{n}{v_{n}}}\left(\hat{f}_{n}(X)-f\right)=F_{n}+\sqrt{\frac{n}{v_{n}}}\left(A_{n}(X)-f\right) .
$$

Remark 3.6. The assumption that $\left|E\left[X_{t} X_{s}\right]\right| \leqslant C|t-s|^{-\beta}$ for some $\beta>1 / 2$ corresponds to the notion of moderating how long the memory of the data might be. For instance, when $X$ represents the discrete-time increments of a process based on fBm with Hurst parameter $H \in(0,1)$, we expect that $\beta=2-2 H$. The restriction $\beta>1 / 2$ means $H<3 / 4$, a well-known threshold for the limit of validity of central limit theorems for quadratic variations of long-memory processes.

In general when $\beta \in\left(0, \frac{1}{2}\right)$, which corresponds to $3 / 4<H<1$, non-central limit theorems can be satisfied. The speed of convergence in these types of scenarios will be slower than when $\beta>1 / 2$, and there is no known general framework to understand whether the speeds which can be obtained in these non-central limit theorems are optimal. See for instance the excellent treatment of the classical Breuer-Major theorem in Nourdin and Peccati (2012, Chapter 7).

The above theorem shows that the speed of convergence in the CLT reaches the so-called Berry-Esséen rate of $1 / \sqrt{n}$ as soon as $\beta>2 / 3$, as long as the terms coming from the bias and variance estimates, namely $\sqrt{n}\left|A_{n}(X)-f\right|$ and $\left|v_{n}-\sigma^{2}\right|$, are no greater than that same order $1 / \sqrt{n}$. The threshold $\beta>2 / 3$ coincides with $H<2 / 3$ 
when one translates into the Hurst-parameter memory scale; this had already been identified for the canonical stationary case of fGn in Biermé et al. (2012), the paper which was the precursor to the optimal fourth moment theorem in Nourdin and Peccati (2015).

\section{Application to Gaussian Ornstein-Uhlenbeck processes}

In this section we will apply the above results to Ornstein-Uhlenbeck processes driven by a Gaussian process which does not necessarily have stationary increments. More precisely we will study the cases which correspond to sub-fractional Brownian motion and to bifractional Brownian motion.

We consider the Gaussian Ornstein-Uhlenbeck process $X=\left\{X_{t}\right\}_{t \geqslant 0}$ defined by the following linear stochastic differential equation

$$
d X_{t}=-\theta X_{t} d t+d G_{t}, \quad X_{0}=0 ;
$$

where $G$ is an arbitrary mean-zero Gaussian process which has Hölder continuous paths of strictly positive order, and $\theta>0$ is an unknown parameter. Our goal is to estimate $\theta$ under the discrete observations $\left\{X_{1}, \ldots, X_{n}\right\}$ as $n \rightarrow \infty$. The equation (4.1) has the following explicit solution

$$
X_{t}=\mathrm{e}^{-\theta t} \int_{0}^{t} \mathrm{e}^{\theta s} d G_{s}, \quad t \geqslant 0
$$

where the integral can be understood in the Young sense, since $\mathrm{e}^{\theta s}$ is a Lipshitz function. The Young sense coincides in this case with Wiener integral sense here. As mentioned in the introduction, we will consider two different cases for $G$ : the sub-fractional Brownian motion and the bifractional Brownian motion.

4.1. Sub-fractional Brownian motion. Consider a sub-fractional Brownian motion $(\mathrm{sfBm}) S^{H}:=\left\{S_{t}^{H}, t \geqslant 0\right\}$ with parameter $H \in(0,1)$ : this is the mean-zero Gaussian process with covariance function

$$
R_{S^{H}}(s, t):=E\left(S_{t}^{H} S_{s}^{H}\right)=t^{2 H}+s^{2 H}-\frac{1}{2}\left((t+s)^{2 H}+|t-s|^{2 H}\right) .
$$

Note that, when $H=\frac{1}{2}, S^{\frac{1}{2}}$ is a standard Brownian motion. By Kolmogorov's continuity criterion and the fact that

$$
E\left(S_{t}^{H}-S_{s}^{H}\right)^{2} \leqslant\left(2-2^{2 H-1}\right)|s-t|^{2 H} ; s, t \geqslant 0,
$$

we deduce that $S^{H}$ has Hölder continuous paths of order $H-\varepsilon$, for every $\varepsilon \in(0, H)$. In this section we replace $G$ given in (4.1) by $S^{H}$. More precisely, we will estimate the drift parameter $\theta$ in the following equation

$$
d X_{t}=-\theta X_{t} d t+d S_{t}^{H}, \quad X_{0}=0
$$

Proposition 4.1. Suppose that $H \in(0,1)$. Set

$$
f_{H}(\theta):=\frac{H \Gamma(2 H)}{\theta^{2 H}} .
$$

Then for every $t>0$,

$$
\left|E\left[X_{t}^{2}\right]-f_{H}(\theta)\right| \leqslant C t^{2 H-2}
$$


Hence

$$
\sqrt{n}\left|A_{n}(X)-f_{H}(\theta)\right| \leqslant \frac{C}{\sqrt{n}} \sum_{i=1}^{n} i^{2 H-2} \leqslant C \begin{cases}n^{-\frac{1}{2}} & \text { if } 0<H<1 / 2 \\ n^{-\frac{1}{2}} \log (n) & \text { if } H=1 / 2 \\ n^{2 H-3 / 2} & \text { if } H>1 / 2 .\end{cases}
$$

In particular, if $0<H<3 / 4$, the hypothesis $(\mathcal{H} 4)$ holds.

Proof: Since $X_{t}=\mathrm{e}^{-\theta t} \int_{0}^{t} \mathrm{e}^{\theta s} d S_{s}^{H}, \quad t \geqslant 0$, we can write (see El Machkouri et al., 2016)

$$
E\left[X_{t}^{2}\right]=\Delta_{H}(t)+\theta I_{2 H}(t)-\frac{\theta^{2}}{2} J_{2 H}(t)
$$

where

$$
\Delta_{H}(t)=2 H e^{-2 \theta t} \int_{0}^{t} s^{2 H-1} e^{\theta s} d s-2 H(2 H-1) e^{-2 \theta t} \int_{0}^{t} d s e^{\theta s} \int_{0}^{s} d r e^{\theta r}(s+r)^{2 H-2},
$$

and

$$
J_{2 H}(t):=\mathrm{e}^{-2 \theta t} \int_{0}^{t} \int_{0}^{t} \mathrm{e}^{\theta s} \mathrm{e}^{\theta r}|s-r|^{2 H} d r d s ; \quad I_{2 H}(t):=\mathrm{e}^{-\theta t} \int_{0}^{t} \mathrm{e}^{\theta s}(t-s)^{2 H} d s .
$$

Hence

$$
\left|E\left[X_{t}^{2}\right]-f_{H}(\theta)\right| \leqslant\left|\Delta_{H}(t)\right|+\theta\left|I_{2 H}(t)-\frac{2 H \Gamma(2 H)}{\theta^{2 H+1}}\right|+\frac{\theta^{2}}{2}\left|J_{2 H}(t)-\frac{2 H \Gamma(2 H)}{\theta^{2 H+2}}\right| .
$$

We will check that each term of the right side of the last inequality is less than $C t^{2 H-2}$. We can write

$$
\Delta_{H}(t)=2 H a_{H}(t)-2 H(2 H-1) b_{H}(t)
$$

where

$$
a_{H}(t):=e^{-2 \theta t} \int_{0}^{t} s^{2 H-1} e^{\theta s} d s ; \quad b_{H}(t):=e^{-2 \theta t} \int_{0}^{t} d s e^{\theta s} \int_{0}^{s} d r e^{\theta r}(s+r)^{2 H-2} .
$$

It is clear that

$$
\left|a_{H}(t)\right| \leqslant \frac{t^{2 H}}{2 H} e^{-\theta t} \leqslant C \mathrm{e}^{-\frac{\theta t}{2}} .
$$

On the other hand,

$$
\begin{aligned}
& \left|b_{H}(t)\right| \\
& =e^{-2 \theta t} \int_{0}^{t} d s e^{\theta s} \int_{0}^{s} d r e^{\theta r}(s+r)^{2 H-2}=\mathrm{e}^{-2 \theta t} \int_{0}^{t} \mathrm{e}^{\theta s} d s \int_{s}^{2 s} \mathrm{e}^{\theta(u-s)} u^{2 H-2} d u \\
& =\mathrm{e}^{-2 \theta t} \int_{0}^{2 t} \mathrm{e}^{\theta u} u^{2 H-2}\left(t \wedge u-\frac{u}{2}\right) d u \\
& =\frac{\mathrm{e}^{-2 \theta t}}{2}\left(\int_{0}^{t} \mathrm{e}^{\theta u} u^{2 H-1} d u+\int_{t}^{2 t}(2 t-u) \mathrm{e}^{\theta u} u^{2 H-2} d u\right) \\
& \leqslant \frac{t^{2 H}}{4 H} \mathrm{e}^{-\theta t}+t^{2 H-2} \int_{t}^{2 t}(2 t-u) \mathrm{e}^{\theta u} d u \leqslant C t^{2 H-2}
\end{aligned}
$$


Hence, for every $t>0,\left|\Delta_{H}(t)\right| \leqslant C t^{2 H-2}$. We also have for $t \geqslant 0$

$$
\begin{aligned}
& \left|J_{2 H}(t)-\frac{\Gamma(2 H+1)}{\theta^{2 H+2}}\right| \\
& =\left|\frac{1}{\theta}\left(\int_{0}^{t} u^{2 H} \mathrm{e}^{-\theta u} d u-\mathrm{e}^{-2 \theta t} \int_{0}^{t} u^{2 H} \mathrm{e}^{\theta u} d u\right)-\frac{\Gamma(2 H+1)}{\theta^{2 H+2}}\right| \\
& \leqslant \frac{1}{\theta}\left|\int_{t}^{\infty} u^{2 H} \mathrm{e}^{-\theta u} d u-\mathrm{e}^{-2 \theta t} \int_{0}^{t} u^{2 H} \mathrm{e}^{\theta u} d u\right| \\
& \leqslant \frac{\mathrm{e}^{-\theta t / 2}}{\theta}\left(\int_{t}^{\infty} u^{2 H} \mathrm{e}^{-\theta u / 2} d u+\int_{0}^{t} u^{2 H} \mathrm{e}^{-\theta u / 2} d u\right) \\
& \leqslant C \mathrm{e}^{-\theta t / 2} .
\end{aligned}
$$

For the last term, let $t \geqslant 0$

$$
\begin{aligned}
\left|I_{2 H}(t)-\frac{\Gamma(2 H+1)}{\theta^{2 H+1}}\right| & =\left|\int_{0}^{t} u^{2 H} \mathrm{e}^{-\theta u} d u-\frac{\Gamma(2 H+1)}{\theta^{2 H+1}}\right| \\
& =\int_{t}^{+\infty} u^{2 H} \mathrm{e}^{-\theta u} d u \leqslant \mathrm{e}^{-\theta t / 2} \int_{t}^{+\infty} \mathrm{e}^{-\theta u / 2} u^{2 H} d u \\
& \leqslant C \mathrm{e}^{-\theta t / 2}
\end{aligned}
$$

which completes the proof.

Proposition 4.2. For every $H \in(0,1)$, the hypothesis $(\mathcal{H} 3)$ holds. More precisely, we have for large $|t-s|$

$$
\left|E\left[X_{t} X_{s}\right]\right| \leqslant C|t-s|^{2 H-2}
$$

Proof: If $H=\frac{1}{2}$, it is easy to see that $\left|E\left[X_{t} X_{s}\right]\right| \leqslant \frac{1}{2 \theta} e^{-\theta(t-s)} \leqslant C|t-s|^{2 H-2}$ for $|t-s|>0$.

Now, suppose that $H \in\left(0, \frac{1}{2}\right) \cup\left(\frac{1}{2}, 1\right)$. Thanks to Lemma 5.2 we get

$$
\begin{aligned}
E\left[X_{t} X_{s}\right]= & \mathrm{e}^{-\theta(t-s)} E\left[X_{s}^{2}\right]+H(2 H-1) \mathrm{e}^{-\theta t} \mathrm{e}^{-\theta s} \int_{s}^{t} d v \mathrm{e}^{\theta v} \int_{0}^{s} d u \mathrm{e}^{\theta u}(v-u)^{2 H-2} \\
& -H(2 H-1) \mathrm{e}^{-\theta t} \mathrm{e}^{-\theta s} \int_{s}^{t} d v \mathrm{e}^{\theta v} \int_{0}^{s} d u \mathrm{e}^{\theta u}(u+v)^{2 H-2}
\end{aligned}
$$

Hence,

$\left|E\left[X_{s} X_{t}\right]\right| \leqslant \mathrm{e}^{-\theta(t-s)} E\left[X_{s}^{2}\right]+2 \mathrm{e}^{-\theta t} \mathrm{e}^{-\theta s} H|2 H-1| \int_{s}^{t} \int_{0}^{s} \mathrm{e}^{\theta u} \mathrm{e}^{\theta v}(v-u)^{2 H-2} d u d v$.

Define for every $\alpha \in(0,1)$

$$
Z_{t}^{\alpha}:=\mathrm{e}^{-\theta t} \int_{-\infty}^{t} \mathrm{e}^{\theta u} d B_{u}^{\alpha}, \quad t \in \mathbb{R}
$$


which is a stationary Gaussian process, where $B^{\alpha}$ is a fractional Brownian motion with Hurst parameter $\alpha \in(0,1)$. Then, we can write for $s<t$,

$$
\begin{aligned}
& H(2 H-1) \mathrm{e}^{-\theta t} \mathrm{e}^{-\theta s} \int_{s}^{t} \int_{0}^{s} \mathrm{e}^{\theta u} \mathrm{e}^{\theta v}(v-u)^{2 H-2} d u d v \\
= & H(2 H-1) \mathrm{e}^{-\theta(t-s)} \int_{0}^{t-s} d y \mathrm{e}^{\theta y} \int_{-s}^{0} d x \mathrm{e}^{\theta x}(y-x)^{2 H-2} \\
= & \mathrm{e}^{-\theta(t-s)} E\left[\left(\int_{0}^{t-s} \mathrm{e}^{\theta y} d B_{y}^{H}\right)\left(\int_{-s}^{0} \mathrm{e}^{\theta x} d B_{x}^{H}\right)\right] \\
= & E\left[\left(Z_{t-s}^{H}-\mathrm{e}^{-\theta(t-s)} Z_{0}^{H}\right)\left(Z_{0}^{H}-\mathrm{e}^{-\theta s} Z_{-s}^{H}\right)\right] \\
= & E\left[Z_{t-s}^{H} Z_{0}^{H}\right]-\mathrm{e}^{-\theta s} E\left[Z_{0}^{H} Z_{t}^{H}\right]-\mathrm{e}^{-\theta(t-s)} E\left[\left(Z_{0}^{H}\right)^{2}\right]+\mathrm{e}^{-\theta t} E\left[Z_{0}^{H} Z_{s}^{H}\right] \\
\leqslant & C\left((t-s)^{2 H-2}+\mathrm{e}^{-\theta(t-s)}\right) .
\end{aligned}
$$

The last inequality comes from the fact that for large $r>0 E\left[Z_{r}^{H} Z_{0}^{H}\right] \leqslant C|r|^{2 H-2}$ (see Cheridito et al., 2003, or Es-Sebaiy and Viens, 2019+).

Define the following rates of convergence,

$$
\varphi_{\alpha}(n)= \begin{cases}n^{-1} & \text { if } 0<\alpha<1 / 2 \\ n^{2 \alpha-2} & \text { if } 1 / 2 \leqslant \alpha<1\end{cases}
$$

and

$$
\psi_{\alpha}(n)= \begin{cases}n^{-1} & \text { if } 0<\alpha<1 / 2 \\ n^{4 \alpha-3} & \text { if } 1 / 2 \leqslant \alpha<3 / 4 .\end{cases}
$$

Proposition 4.3. Let $0<H<3 / 4$. Define

$$
\sigma_{H}^{2}:=2 \rho_{H}(0)^{2}+4 \sum_{i \in \mathbb{N} \backslash\{0\}} \rho_{H}(i)^{2}
$$

where $\rho_{H}(k):=E\left[Z_{k}^{H} Z_{0}^{H}\right], k \in \mathbb{N}$ and $Z$ is the process given in (4.4). Note that $\rho_{H}(0)=f_{H}(\theta)$. Then

$$
\left|E\left[V_{n}(X)^{2}\right]-\sigma_{H}^{2}\right| \leqslant C \psi_{H}(n) .
$$

In particular, the hypothesis $(\mathcal{H} 2)$ holds.

If $H=3 / 4$, we have

$$
\left|\frac{E\left[V_{n}(X)^{2}\right]}{\log (n)}-\frac{9}{16 \theta^{4}}\right| \leqslant C \log (n)^{-1} .
$$

Proof: See Appendix.

Propositions 4.1 and 4.3 lead to the assumptions $(\mathcal{H} 1)$ and $(\mathcal{H} 2)$. Then, applying Theorem 3.1 we obtain the strong consistency of the estimator $\hat{f}_{n}(X)$ of the form (3.1). 
Theorem 4.4. Let $0<H<1$. Then we have

$$
\hat{f}_{n}(X) \longrightarrow f_{H}(\theta)=\frac{H \Gamma(2 H)}{\theta^{2 H}}
$$

almost surely as $n \longrightarrow \infty$.

Now, we will study the asymptotic normality of the estimator $\hat{f}_{n}(X)$ when $0<H \leqslant \frac{3}{4}$. Using (3.6) with $\beta=2-2 H$ we obtain the following result.

Proposition 4.5. If $0<H \leqslant \frac{3}{4}$, then

$$
d_{T V}\left(F_{n}(X), N\right) \leqslant C \begin{cases}n^{-\frac{1}{2}} & \text { if } H \in\left(0, \frac{2}{3}\right) \\ n^{-\frac{1}{2}} \log (n)^{2} & \text { if } H=\frac{2}{3} \\ n^{6 H-\frac{9}{2}} & \text { if } H \in\left(\frac{2}{3}, \frac{3}{4}\right) \\ \log (n)^{-3 / 2} & \text { if } H=\frac{3}{4} .\end{cases}
$$

Combining this with Propositions 4.1 and 4.3 we deduce the result.

Theorem 4.6. If $0<H<3 / 4$, then

$$
d_{T V}\left(\frac{\sqrt{n}}{\sigma_{H}}\left(\hat{f}_{n}(X)-f_{H}(\theta)\right), N\right) \leqslant C \sqrt{\psi_{H}(n)}
$$

and if $H=3 / 4$, we have

$$
d_{T V}\left(\frac{\sqrt{n}\left(\hat{f}_{n}(X)-f_{H}(\theta)\right)}{\sigma_{H} \sqrt{\log (n)}}, N\right) \leqslant C \log (n)^{-1 / 2} .
$$

In particular, if $0<H<3 / 4$, then, as $n \rightarrow \infty$

$$
\sqrt{n}\left(\hat{f}_{n}(X)-f_{H}(\theta)\right) \stackrel{\text { law }}{\longrightarrow} \mathcal{N}\left(0, \sigma_{H}^{2}\right)
$$

and if $H=3 / 4$, then, as $n \rightarrow \infty$

$$
\frac{\sqrt{n}\left(\hat{f}_{n}(X)-f_{H}(\theta)\right)}{\sqrt{\log (n)}} \stackrel{\text { law }}{\longrightarrow} \mathcal{N}\left(0, \sigma_{H}^{2}\right) .
$$

4.2. Bifractional Brownian motion. In this section we suppose that $G$ given in (4.1) is a bifractional Brownian motion (bifBm) $B^{H, K}$ with parameters $H \in(0,1)$ and $K \in(0,1]$. The $B^{H, K}:=\left\{B_{t}^{H, K}, t \geqslant 0\right\}$ is the mean-zero Gaussian process with covariance function

$$
E\left(B_{s}^{H, K} B_{t}^{H, K}\right)=\frac{1}{2^{K}}\left(\left(t^{2 H}+s^{2 H}\right)^{K}-|t-s|^{2 H K}\right) .
$$

The case $K=1$ corresponds to the fBm with Hurst parameter $H$. The process $B^{H, K}$ verifies,

$$
E\left(\left|B_{t}^{H, K}-B_{s}^{H, K}\right|^{2}\right) \leqslant 2^{1-K}|t-s|^{2 H K}
$$

so $B^{H, K}$ has $(H K-\varepsilon)-$ Hölder continuous paths for any $\varepsilon \in(0, H K)$ thanks to Kolmogorov's continuity criterion. 
Proposition 4.7. Assume that $H \in(0,1)$ and $K \in(0,1]$. Then we have for large $t>0$

$$
\left|E\left[X_{t}^{2}\right]-f_{H, K}(\theta)\right| \leqslant C t^{2 H K-2}
$$

where

$$
f_{H, K}(\theta):=2^{1-K} H K \Gamma(2 H K) / \theta^{2 H K}
$$

Then

$$
\sqrt{n}\left|A_{n}(X)-\mu(\theta)\right| \leqslant C \begin{cases}n^{-\frac{1}{2}} & \text { if } 0<H K<1 / 2 \\ n^{2 H K-3 / 2} & \text { if } H K \geqslant 1 / 2 .\end{cases}
$$

In particular, if $H K<3 / 4$, the hypothesis (H4) holds.

Proof: From El Machkouri et al. (2016) and the fact that $X_{t}=\mathrm{e}^{-\theta t} \int_{0}^{t} \mathrm{e}^{\theta s} d B_{s}^{H, K}, t \geqslant 0$, we can write

$$
E\left[X_{t}^{2}\right]=\Delta_{H, K}(t)+2^{1-K} \theta I_{2 H K}(t)-2^{-K} \theta^{2} J_{2 H K}(t),
$$

where

$$
\begin{aligned}
& \Delta_{H, K}(t) \\
& =2^{2-K} H K \mathrm{e}^{-2 \theta t} \int_{0}^{t} \mathrm{e}^{\theta s} s^{2 H K-1} d s \\
& +2^{3-K} H^{2} K(K-1) \mathrm{e}^{-2 \theta t} \int_{0}^{t} \int_{0}^{s}(s r)^{2 H-1}\left(s^{2 H}+r^{2 H}\right)^{K-2} \mathrm{e}^{\theta r} \mathrm{e}^{\theta s} d r d s
\end{aligned}
$$

and

$J_{2 H K}(t)=\mathrm{e}^{-2 \theta t} \int_{0}^{t} \int_{0}^{s} \mathrm{e}^{\theta s} \mathrm{e}^{\theta r}(s-r)^{2 H K} d r d s ; \quad I_{2 H K}(t)=\mathrm{e}^{-\theta t} \int_{0}^{t} \mathrm{e}^{\theta s}(t-s)^{2 H K} d s$.

Hence

$$
\begin{aligned}
\left|E\left[X_{t}^{2}\right]-\mu(\theta)\right| \leqslant & \left|\Delta_{H}(t)\right|+\frac{\theta}{2^{K-1}}\left|I_{2 H K}(t)-\frac{2 H K \Gamma(2 H K)}{\theta^{2 H K+1}}\right| \\
& +\frac{\theta^{2}}{2^{K}}\left|J_{2 H K}(t)-\frac{2 H K \Gamma(2 H K)}{\theta^{2 H K+2}}\right| .
\end{aligned}
$$

We will check that each term of the right-hand side is less than $C t^{2 H K-2}$. We can write

$$
\Delta_{H, K}(t)=2^{2-K} H K a_{H, K}(t)+2^{3-K} H^{2} K(K-1) b_{H, K}(t) .
$$

where

$$
a_{H, K}(t):=\mathrm{e}^{-2 \theta t} \int_{0}^{t} \mathrm{e}^{\theta s} s^{2 H K-1} d s
$$

and

$$
b_{H, K}(t):=\mathrm{e}^{-2 \theta t} \int_{0}^{t} \int_{0}^{s}(s r)^{2 H-1}\left(s^{2 H}+r^{2 H}\right)^{K-2} \mathrm{e}^{\theta r} \mathrm{e}^{\theta s} d r d s .
$$

It is easy to prove that

$$
a_{H, K}(t) \leqslant C \mathrm{e}^{-\theta t / 2}
$$


On the other hand, using $x^{2}+y^{2} \geqslant 2|x y|, x, y \in \mathbb{R}$, we get

$$
\begin{aligned}
b_{H, K}(t) & =\mathrm{e}^{-2 \theta t} \int_{0}^{t} \int_{0}^{s}(s r)^{2 H-1}\left(s^{2 H}+r^{2 H}\right)^{K-2} \mathrm{e}^{\theta r} \mathrm{e}^{\theta s} d r d s \\
& \leqslant \mathrm{e}^{-2 \theta t} 2^{K-2} \int_{0}^{t} \int_{0}^{s}(s r)^{2 H-1} s^{H K-1} r^{H K-1} \mathrm{e}^{\theta r} \mathrm{e}^{\theta s} d r d s \\
& :=b_{1}(t)+b_{2}(t)
\end{aligned}
$$

where

$$
\begin{aligned}
& b_{1}(t)=\mathrm{e}^{-2 \theta t} \int_{0}^{\frac{t}{2}} \int_{0}^{s}(s r)^{H K-1} \mathrm{e}^{\theta r} \mathrm{e}^{\theta s} d r d s, \\
& b_{2}(t)=\mathrm{e}^{-2 \theta t} \int_{\frac{t}{2}}^{t} \int_{0}^{s}(s r)^{H K-1} \mathrm{e}^{\theta r} \mathrm{e}^{\theta s} d r d s .
\end{aligned}
$$

It is easy to see that

$$
\left|b_{1}(t)\right| \leqslant C t^{2 H K} \mathrm{e}^{-\theta t} \leqslant C \mathrm{e}^{-\frac{\theta t}{2}},
$$

and

$$
\begin{aligned}
\left|b_{2}(t)\right| & \leqslant\left(\frac{t}{2}\right)^{H K-1} \frac{\mathrm{e}^{-\theta t}}{\theta} \int_{0}^{t} r^{H K-1} \mathrm{e}^{\theta r} d r \\
& \leqslant\left(\frac{t}{2}\right)^{H K-1} \frac{\mathrm{e}^{-\theta t}}{\theta}\left(\int_{0}^{\frac{t}{2}} r^{H K-1} \mathrm{e}^{\theta r} d r+\int_{\frac{t}{2}}^{t} r^{H K-1} \mathrm{e}^{\theta r} d r\right) \\
& \leqslant\left(\frac{t}{2}\right)^{2 H K-1} \frac{e^{-\theta t / 2}}{\theta}+\frac{1}{\theta^{2}}\left(\frac{t}{2}\right)^{2 H K-2} \\
& \leqslant C t^{2 H K-2}
\end{aligned}
$$

We deduce that

$$
\Delta_{H, K}(t) \leqslant C t^{2 H K-2}
$$

Moreover, by a similar argument as in the proof of Proposition 4.1 we have

$$
\left|I_{2 H K}(t)-\frac{2 H K \Gamma(2 H K)}{\theta^{2 H K+1}}\right| \leqslant e^{-\theta t / 2}\left(\frac{2}{\theta}\right)^{2 H K+1} \Gamma(2 H K+1)
$$

and

$$
\left|J_{2 H K}(t)-\frac{2 H K \Gamma(2 H K)}{\theta^{2 H K+2}}\right| \leqslant\left(\frac{2}{\theta}\right)^{2 H K+2} \Gamma(2 H K+1) \mathrm{e}^{-\theta t / 2}
$$

which completes the proof.

Proposition 4.8. For all fixed $(H, K) \in(0,1) \times(0,1]$, with $H K \neq \frac{1}{2}$, the hypothesis $(\mathcal{H} 3)$ holds. More precisely, we have for large $|t-s|$,

$$
\left|E\left[X_{t} X_{s}\right]\right| \leqslant C \begin{cases}|t-s|^{2 H K-2 H-1} & \text { if } 0<H K<1 / 2 \\ |t-s|^{2 H K-2} & \text { if } 1 / 2<H K<1 .\end{cases}
$$


Proof: Let $s<t$. Using Lemma 5.2 we get

$$
\begin{aligned}
E & {\left[X_{t} X_{s}\right] } \\
= & \mathrm{e}^{-\theta(t-s)} E\left[X_{s}^{2}\right] \\
& -2^{2-K} K(1-K) \mathrm{e}^{-\theta t} \mathrm{e}^{-\theta s} \int_{s}^{t} \mathrm{e}^{\theta v} \int_{0}^{s} \mathrm{e}^{\theta u}(u v)^{2 H-1}\left(u^{2 H}+v^{2 H}\right)^{K-2} d u d v \\
& +2^{1-K} H K(2 H K-1) \mathrm{e}^{-\theta t} \mathrm{e}^{-\theta s} \int_{s}^{t} \mathrm{e}^{\theta v} \int_{0}^{s} \mathrm{e}^{\theta u}(u-v)^{2 H K-2} d u d v .
\end{aligned}
$$

As in the proof of Proposition 4.2 we have

$$
\delta_{H K}:=e^{-\theta t} \mathrm{e}^{-\theta s} \int_{s}^{t} \mathrm{e}^{\theta v} \int_{0}^{s} \mathrm{e}^{\theta u}(u-v)^{2 H K-2} d u d v \leqslant C|t-s|^{2 H K-2} .
$$

Set

$$
\lambda_{H, K}:=\mathrm{e}^{-\theta t} \mathrm{e}^{-\theta s} \int_{s}^{t} \mathrm{e}^{\theta v} \int_{0}^{s} \mathrm{e}^{\theta u}(u v)^{2 H-1}\left(u^{2 H}+v^{2 H}\right)^{K-2} d u d v .
$$

If $H \geqslant \frac{1}{2}$, we have for $0 \leqslant u \leqslant v$,

$$
(u v)^{2 H-1}\left(u^{2 H}+v^{2 H}\right)^{K-2} \leqslant v^{2 H K-2} \leqslant(v-u)^{2 H K-2} .
$$

Thus, if we assume that $H K \neq \frac{1}{2}, \lambda_{H, K} \leqslant C \delta_{H K} \leqslant C|t-s|^{2 H K-2}$.

If $H<\frac{1}{2}$, it is clear that $s \rightarrow \mathrm{e}^{-\theta s} \int_{0}^{s} \mathrm{e}^{\theta u} u^{2 H-1} d u$ is bounded. This implies

$$
\lambda_{H, K} \leqslant C \mathrm{e}^{-\theta t} \int_{s}^{t} \mathrm{e}^{\theta v} v^{2 H-1} v^{2 H K-4 H} d v \leqslant C t^{2 H K-2 H-1} \leqslant C(t-s)^{2 H K-2 H-1}
$$

which finishes the proof.

Using the same arguments as in Proposition 4.3 we obtain the following result.

Proposition 4.9. For all fixed $0<H K<3 / 4$,

$$
\left|E\left[V_{n}(X)^{2}\right]-\sigma_{H, K}^{2}\right| \leqslant C \psi_{H K}(n)
$$

where

$$
\sigma_{H, K}^{2}:=4 \sum_{i \in \mathbb{N}^{*}}\left(\rho_{H, K}(i)-\left(1-2^{1-K}\right) \mathrm{e}^{-\theta i} \rho_{H, K}(0)\right)^{2}+2^{3-2 K} \rho_{H, K}(0)^{2}
$$

with $\rho_{H, K}(k):=E\left[Z_{k}^{H K} Z_{0}^{H K}\right], k \in \mathbb{N}$ where $Z^{H K}$ is the process given in (4.4), and $\rho_{H, K}(0)=\frac{H K \Gamma(2 H K)}{\theta^{2 H K}}$. In particular, the hypothesis $(\mathcal{H} 2)$ holds.

If $H K=3 / 4$, we have

$$
\left|\frac{E\left[V_{n}(X)^{2}\right]}{\log (n)}-\frac{9}{16 \theta^{4}}\right| \leqslant C \log (n)^{-1} .
$$

Similarly as in Subsection 4.1 we obtain the following asymptotic behavior results.

Theorem 4.10. Let $H, K \in(0,1)$. Then we have

$$
\hat{f}_{n}(X) \longrightarrow f_{H, K}(\theta)=2^{1-K} H K \Gamma(2 H K) / \theta^{2 H K}
$$

almost surely as $n \longrightarrow \infty$. 
Theorem 4.11. Let $H K \in(0,3 / 4) \backslash\{1 / 2\}$ and $N \sim \mathcal{N}(0,1)$, then

$$
d_{T V}\left(\sqrt{n}\left(\hat{f}_{n}(X)-f_{H, K}(\theta)\right) / \sigma_{H K}, N\right) \leqslant C \sqrt{\psi_{H K}(n)}
$$

and if $H K=3 / 4$, we have

$$
d_{T V}\left(\frac{\sqrt{n}\left(\hat{f}_{n}(X)-f_{H, K}(\theta)\right)}{\sigma_{H K} \sqrt{\log (n)}}, N\right) \leqslant C \log (n)^{-1 / 2} .
$$

In particular, if $H K \in(0,3 / 4) \backslash\{1 / 2\}$, we have as $n \rightarrow \infty$

$$
\sqrt{n}\left(\hat{f}_{n}(X)-f_{H, K}(\theta)\right) \stackrel{\text { law }}{\longrightarrow} \mathcal{N}\left(0, \sigma_{H, K}^{2}\right)
$$

and if $H K=3 / 4$, we have as $n \rightarrow \infty$

$$
\frac{\sqrt{n}\left(\hat{f}_{n}(X)-f_{H, K}(\theta)\right)}{\sqrt{\log (n)}} \stackrel{\text { law }}{\longrightarrow} \mathcal{N}\left(0, \sigma_{H, K}^{2}\right) .
$$

\section{Appendix}

Lemma 5.1. Let $N \sim \mathcal{N}(0,1), \mu \in \mathbb{R}$ and $\sigma>0$. Then

$$
d_{T V}(\mu+\sigma N, N) \leqslant \sqrt{\frac{\pi}{2}}|\mu|+2\left|1-\sigma^{2}\right| .
$$

Consequently, for every integrable real-valued random variable $F$,

$$
d_{T V}(\mu+\sigma F, N) \leqslant d_{T V}(F, N)+\sqrt{\frac{\pi}{2}}|\mu|+2\left|1-\frac{1}{\sigma^{2}}\right| .
$$

Proof: In order to prove this lemma we will need the following result: for every bounded function $f \in \mathcal{C}^{1}(\mathbb{R})$, we have

$$
E(N f(\mu+\sigma N))=\sigma E\left(f^{\prime}(\mu+\sigma N)\right) .
$$

Indeed, by integration by parts, straightforward calculation leads to

$$
\begin{aligned}
E(N f(\mu+\sigma N)) & =\int_{-\infty}^{\infty} x f(\mu+\sigma x) \frac{e^{-x^{2} / 2}}{\sqrt{2 \pi}} d x=\sigma \int_{-\infty}^{\infty} f^{\prime}(\mu+\sigma x) \frac{e^{-x^{2} / 2}}{\sqrt{2 \pi}} d x \\
& =\sigma E\left(f^{\prime}(\mu+\sigma N)\right) .
\end{aligned}
$$

Thus, using (2.4), we can conclude

$$
\begin{aligned}
& d_{T V}(\mu+\sigma N, N) \\
& =\sup _{f \in \mathcal{C}^{1}(\mathbb{R}),\|f\|_{\infty} \leq \sqrt{\pi / 2},\left\|f^{\prime}\right\|_{\infty} \leq 2}\left|E\left(f^{\prime}(\mu+\sigma N)-(\mu+\sigma N) f(\mu+\sigma N)\right)\right| \\
& =\sup _{f \in \mathcal{C}^{1}(\mathbb{R}),\|f\|_{\infty} \leq \sqrt{\pi / 2},\left\|f^{\prime}\right\|_{\infty} \leq 2}\left|\left(1-\sigma^{2}\right) E f^{\prime}(\mu+\sigma N)-\mu E f(\mu+\sigma N)\right| \\
& \leqslant \sqrt{\frac{\pi}{2}}|\mu|+2\left|1-\sigma^{2}\right|,
\end{aligned}
$$


which proves (5.1).

Now, let us prove (5.2). If $f \in \mathcal{C}^{1}(\mathbb{R}),\|f\|_{\infty} \leq \sqrt{\pi / 2}$ and $\left\|f^{\prime}\right\|_{\infty} \leq 2$, we have

$$
\begin{aligned}
& \left|E\left(f^{\prime}(\mu+\sigma F)-(\mu+\sigma F) f(\mu+\sigma F)\right)\right| \\
& \leqslant\left|E\left(f^{\prime}(\mu+\sigma F)-\sigma F f(\mu+\sigma F)\right)\right|+|\mu E f(\mu+\sigma F)| \\
& \leqslant d_{T V}(\sigma F, N)+\sqrt{\pi / 2}|\mu|,
\end{aligned}
$$

where we used that $x \longrightarrow f(\mu+x) \in \mathcal{C}^{1}(\mathbb{R}),\|f(\mu+.)\|_{\infty} \leq \sqrt{\pi / 2}$ and $\|(f(\mu+$ .) $)^{\prime} \|_{\infty} \leq 2$.

Moreover,

$$
\begin{aligned}
d_{T V}(\sigma F, N) & =d_{T V}\left(F, \frac{1}{\sigma} N\right) \\
& \leqslant d_{T V}(F, N)+d_{T V}\left(N, \frac{1}{\sigma} N\right) \\
& \leqslant d_{T V}(F, N)+2\left|1-\frac{1}{\sigma^{2}}\right|,
\end{aligned}
$$

where the last inequality comes from (5.1). We can then conclude (5.2).

Lemma 5.2. Let $G$ be a Gaussian process which has Hölder continuous paths of strictly positive order and $X$ is the solution of the equation (4.1). Define $R_{G}(s, t)=$ $E\left[G_{s} G_{t}\right]$, and assume that $\frac{\partial R_{G}}{\partial s}(s, r)$ and $\frac{\partial^{2} R_{G}}{\partial s \partial r}(s, r)$ exist on $(0, \infty) \times(0, \infty)$. Then for every $0<s<t$, we have

$$
E\left[X_{s} X_{t}\right]=\mathrm{e}^{-\theta(t-s)} E\left[X_{s}^{2}\right]+\mathrm{e}^{-\theta t} \mathrm{e}^{-\theta s} \int_{s}^{t} \mathrm{e}^{\theta v} \int_{0}^{s} \mathrm{e}^{\theta u} \frac{\partial^{2} R_{G}}{\partial u \partial v}(u, v) d u d v .
$$

Proof: Fix $s<t$. We have

$$
\begin{aligned}
E\left[X_{s} X_{t}\right] & =E\left[\left(\mathrm{e}^{-\theta s} \int_{0}^{s} \mathrm{e}^{\theta u} d G_{u}\right)\left(\mathrm{e}^{-\theta t} \int_{0}^{t} \mathrm{e}^{\theta v} d G_{v}\right)\right] \\
& =\mathrm{e}^{-\theta(t-s)} E\left[X_{s}^{2}\right]+\mathrm{e}^{-\theta t} \mathrm{e}^{-\theta s} E\left[\int_{0}^{s} \mathrm{e}^{\theta u} d G_{u} \int_{s}^{t} \mathrm{e}^{\theta v} d G_{v}\right] .
\end{aligned}
$$

Moreover, by (2.1),

$$
\begin{aligned}
& \mathrm{e}^{-\theta t} \mathrm{e}^{-\theta s} E\left[\int_{0}^{s} \mathrm{e}^{\theta u} d G_{u} \int_{s}^{t} \mathrm{e}^{\theta v} d G_{v}\right] \\
& =\mathrm{e}^{-\theta t} \mathrm{e}^{-\theta s} E\left[\left(\mathrm{e}^{\theta s} G_{s}-\theta \int_{0}^{s} \mathrm{e}^{\theta u} G_{u} d u\right)\left(\mathrm{e}^{\theta t} G_{t}-\mathrm{e}^{\theta s} G_{s}^{H}-\theta \int_{s}^{t} \mathrm{e}^{\theta v} G_{v} d v\right)\right] \\
& =\mathrm{e}^{-\theta t} \mathrm{e}^{-\theta s}\left[\mathrm{e}^{\theta(t+s)} R_{G}(s, t)-\mathrm{e}^{2 \theta s} R_{G}(s, s)\right. \\
& -\theta \mathrm{e}^{\theta s} \int_{s}^{t} \mathrm{e}^{\theta v} R_{G}(s, v) d v-\theta \mathrm{e}^{\theta t} \int_{0}^{s} \mathrm{e}^{\theta u} R_{G}(u, t) d u \\
& \left.+\theta \mathrm{e}^{\theta s} \int_{0}^{s} \mathrm{e}^{\theta u} R_{G}(u, s) d u+\theta^{2} \int_{s}^{t} \int_{0}^{s} \mathrm{e}^{\theta u} \mathrm{e}^{\theta v} R_{G}(u, v) d u d v\right] .
\end{aligned}
$$

Applying again (2.1) several times we get the last term of (5.3). Thus the desired result is obtained. 
Lemma 5.3. Let $X$ be the solution of (4.2), and let $\sigma_{H}^{2}$ be the constant defined in Proposition 4.3. If $0<H<3 / 4$, we have

$$
\left|E\left[V_{n}(X)^{2}\right]-\sigma_{H}^{2}\right| \leqslant C \psi_{H}(n)
$$

If $H=3 / 4$, we have

$$
\left|\frac{E\left[V_{n}(X)^{2}\right]}{\log (n)}-\frac{9}{16 \theta^{4}}\right| \leqslant C \log (n)^{-1} .
$$

Proof: We have $V_{n}(X)=I_{2}\left(f_{n, 2}\right)$, with $f_{n, 2}=\frac{1}{\sqrt{n}} \sum_{i=1}^{n} f_{i}^{\otimes 2}$. Then

$$
\begin{aligned}
E\left[V_{n}(X)^{2}\right] & =\frac{2}{n} \sum_{k, l=1}^{n}\left(\left\langle f_{k}, f_{l}\right\rangle_{\mathcal{H}}\right)^{2}=\frac{2}{n} \sum_{k, l=1}^{n}\left(E\left[X_{k} X_{l}\right]\right)^{2} \\
& =\frac{2}{n} \sum_{k=1}^{n}\left(E\left[X_{k}^{2}\right]\right)^{2}+\frac{2}{n} \sum_{\substack{k, l=1 \\
k \neq l}}^{n}\left(E\left[X_{k} X_{j}\right]\right)^{2} .
\end{aligned}
$$

We will need the following lemmas.

Lemma 5.4. Let $X$ be the solution of (4.2). Then,

$$
\left|\frac{1}{n} \sum_{k=1}^{n}\left(E\left[X_{k}^{2}\right]\right)^{2}-f_{H}(\theta)^{2}\right| \leqslant C \varphi_{H}(n)
$$

Proof: By Proposition 4.1 we deduce that

$$
\begin{aligned}
\left|\frac{1}{n} \sum_{k=1}^{n}\left(E\left[X_{k}^{2}\right]\right)^{2}-f_{H}(\theta)^{2}\right| & \leqslant \frac{1}{n} \sum_{k=1}^{n}\left|E\left[X_{k}^{2}\right]-f_{H}(\theta)\right|\left(E\left[X_{k}^{2}\right]+f_{H}(\theta)\right) \\
& \leqslant \frac{C}{n} \sum_{k=1}^{n}\left|E\left[X_{k}^{2}\right]-f_{H}(\theta)\right| \leqslant \frac{C}{n} \sum_{k=1}^{n} k^{2 H-2} \\
& \leqslant C \varphi_{H}(n) .
\end{aligned}
$$

Lemma 5.5. Let $X$ be the solution of (4.2). If $0<H<3 / 4$, we have

$$
J(n):=\left|\frac{1}{n} \sum_{\substack{j, k=1 \\ k \neq j}}^{n}\left(E\left[X_{k} X_{j}\right]\right)^{2}-2 \sum_{i \in \mathbb{N}^{*}} \rho_{H}(i)^{2}\right| \leqslant C \psi_{H}(n) .
$$

Proof: Suppose that $H \neq 1 / 2$. Using (5.3), we have

$$
\frac{1}{n} \sum_{k, j=1}^{n}\left(E\left[X_{k} X_{j}\right]\right)^{2}=E_{1}(n)+E_{2}(n)+E_{3}(n)+E_{4}(n)+E_{5}(n)+E_{6}(n)
$$

where

$$
E_{1}(n):=\frac{2}{n} \sum_{j=1}^{n-1} \sum_{k=j+1}^{n} a(k, j)^{2}, \quad E_{2}(n):=\frac{4}{n} \sum_{j=1}^{n-1} \sum_{k=j+1}^{n} a(k, j) A(k, j)
$$




$$
\begin{aligned}
& E_{3}(n):=\frac{4}{n} \sum_{j=1}^{n-1} \sum_{k=j+1}^{n} a(k, j) D(k, j), \quad E_{4}(n):=\frac{2}{n} \sum_{j=1}^{n-1} \sum_{k=j+1}^{n} A(k, j)^{2} \\
& E_{5}(n):=\frac{4}{n} \sum_{j=1}^{n-1} \sum_{k=j+1}^{n} A(k, j) D(k, j), \quad E_{6}(n):=\frac{2}{n} \sum_{j=1}^{n-1} \sum_{k=j+1}^{n} D(k, j)^{2}
\end{aligned}
$$

with for every $s \leqslant t$

$$
\begin{gathered}
a(s, t):=\mathrm{e}^{-\theta(t-s)} E\left[X_{s}^{2}\right], \\
A(t, s):=H(2 H-1) \mathrm{e}^{-\theta t} \mathrm{e}^{-\theta s} \int_{s}^{t} \mathrm{e}^{\theta v} \int_{0}^{s} \mathrm{e}^{\theta u}(u-v)^{2 H-2} d u d v, \\
D(t, s):=-H(2 H-1) \mathrm{e}^{-\theta t} \mathrm{e}^{-\theta s} \int_{s}^{t} \mathrm{e}^{\theta v} \int_{0}^{s} \mathrm{e}^{\theta u}(u+v)^{2 H-2} d u d v .
\end{gathered}
$$

Then, we can write

$$
\begin{aligned}
J(n) \leqslant & \left|E_{1}(n)-\frac{2 f_{H}(\theta)^{2} \mathrm{e}^{-2 \theta}}{1-\mathrm{e}^{-2 \theta}}\right|+\left|E_{2}(n)-4 f_{H}(\theta)\left(\sum_{i=1}^{\infty} \mathrm{e}^{-\theta i} \rho(i)-\frac{f_{H}(\theta) e^{-2 \theta}}{1-\mathrm{e}^{-2 \theta}}\right)\right| \\
& +\left|E_{3}(n)\right|+\left|E_{4}(n)-2 \sum_{i=1}^{\infty}\left(\rho(i)-f_{H}(\theta) \mathrm{e}^{-\theta i}\right)^{2}\right|+\left|E_{5}(n)\right|+\left|E_{6}(n)\right| .
\end{aligned}
$$

On the other hand,

$$
\begin{aligned}
\mid E_{1}(n)-\frac{2 f_{H}(\theta)^{2} \mathrm{e}^{-2 \theta}}{1-\mathrm{e}^{-2 \theta} \mid \leqslant} & \frac{2}{n} \sum_{j=1}^{n-1} \sum_{k=j+1}^{n} \mathrm{e}^{-2 \theta(k-j)}\left(\left[E\left(X_{j}^{2}\right)\right]^{2}-f_{H}(\theta)^{2}\right) \\
& +\left|\frac{2}{n} \sum_{j=1}^{n-1} \sum_{k=j+1}^{n} \mathrm{e}^{-2 \theta(k-j)} f_{H}(\theta)^{2}-\frac{2 f_{H}(\theta)^{2} \mathrm{e}^{-2 \theta}}{1-\mathrm{e}^{-2 \theta}}\right| \\
\leqslant & \frac{2 C}{n} \sum_{j=1}^{n-1} \sum_{k=j+1}^{n} \mathrm{e}^{-2 \theta(k-j)} j^{2 H-2} \\
& +2 f_{H}(\theta)^{2}\left(\sum_{i=n}^{\infty} e^{-2 \theta i}+\frac{1}{n} \sum_{i=1}^{n-1} i \mathrm{e}^{-2 \theta i}\right) \\
\leqslant & C\left[\frac{1}{n} \sum_{j=1}^{n-1} j^{2 H-2} \sum_{i=1}^{n-j} e^{-2 \theta i}+e^{-2 \theta n}+n^{-1}\right] \\
\leqslant & C \varphi_{H}(n) .
\end{aligned}
$$


Furthermore,

$$
\begin{aligned}
E_{2}(n)= & \frac{4}{n} \sum_{j=1}^{n-1} \sum_{k=j+1}^{n} \mathrm{e}^{-\theta(k-j)} E\left(X_{j}^{2}\right)\left[E\left[Z_{k-j} Z_{0}\right]-\mathrm{e}^{-\theta(k-j)} E\left[Z_{0}^{2}\right]\right. \\
& \left.-\mathrm{e}^{-\theta j} E\left[Z_{0} Z_{k}\right]+\mathrm{e}^{-\theta k} E\left[Z_{0} Z_{j}\right]\right] \\
= & \frac{4}{n} \sum_{j=1}^{n-1} \sum_{k=j+1}^{n} \mathrm{e}^{-\theta(k-j)} E\left(X_{j}^{2}\right) \rho(k-j)-\frac{4 \rho(0)}{n} \sum_{j=1}^{n-1} \sum_{k=j+1}^{n} \mathrm{e}^{-2 \theta(k-j)} E\left(X_{j}^{2}\right) \\
& -\frac{4}{n} \sum_{j=1}^{n-1} \mathrm{e}^{-\theta j} E\left(X_{j}^{2}\right) \sum_{k=j+1}^{n} \mathrm{e}^{-\theta(k-j)} \rho(k) \\
& +\frac{4}{n} \sum_{j=1}^{n-1} \rho(j) E\left(X_{j}^{2}\right) \sum_{k=j+1}^{n} \mathrm{e}^{-\theta(k-j)} \mathrm{e}^{-\theta k} \\
:= & E_{2}^{1}(n)-E_{2}^{2}(n)-E_{2}^{3}(n)+E_{2}^{4}(n) .
\end{aligned}
$$

For $E_{2}^{1}(n)$, we have

$$
\begin{aligned}
& \left|E_{2}^{1}(n)-4 f_{H}(\theta) \sum_{i \in \mathbb{N}^{*}} \mathrm{e}^{-\theta i} \rho(i)\right| \\
& \leqslant \frac{4}{n} \sum_{j=1}^{n-1} \sum_{k=j+1}^{n} \mathrm{e}^{-\theta(k-j)}\left|E\left(X_{j}^{2}\right)-f_{H}(\theta)\right||\rho(k-j)| \\
& \quad+4\left|f_{H}(\theta)\right| \frac{1}{n} \sum_{j=1}^{n-1} \sum_{k=j+1}^{n} \mathrm{e}^{-\theta(k-j)} \rho(k-j)-\sum_{i=1}^{\infty} \mathrm{e}^{-\theta i} \rho(i) \mid \\
& \leqslant C\left(n^{2 H-2}+n^{-1}\right)
\end{aligned}
$$

because

$$
\begin{aligned}
\left|\frac{1}{n} \sum_{j=1}^{n-1} \sum_{k=j+1}^{n} \mathrm{e}^{-\theta(k-j)} \rho(k-j)-\sum_{i=1}^{\infty} \mathrm{e}^{-\theta i} \rho(i)\right| & =\left|\sum_{i=n}^{\infty} \mathrm{e}^{-\theta i} \rho(i)-\frac{1}{n} \sum_{i=1}^{n-1} i \mathrm{e}^{-\theta i} \rho(i)\right| \\
& \leqslant \sum_{i=n}^{\infty} \mathrm{e}^{-\theta i}|\rho(i)|+\frac{1}{n} \sum_{i=1}^{n-1} i|\rho(i)| \mathrm{e}^{-\theta i} \\
& \leqslant C\left(n^{2 H-2}+n^{-1}\right)
\end{aligned}
$$

and by using a similar argument as in above, we also have

$$
\frac{4}{n} \sum_{j=1}^{n-1} \sum_{k=j+1}^{n} \mathrm{e}^{-\theta(k-j)}\left|E\left(X_{j}^{2}\right)-f_{H}(\theta)\right||\rho(k-j)| \leqslant C\left(n^{2 H-2}+n^{-1}\right) .
$$

By straightforward calculus, we also have

$$
\left|E_{2}^{2}(n)-4 f_{H}(\theta) \sum_{i=1}^{\infty} \mathrm{e}^{-2 \theta i}\right| \leqslant C \varphi_{H}(n) .
$$


For $E_{2}^{3}(n)$, we have

$$
\begin{aligned}
\left|E_{2}^{3}(n)\right| \leqslant & \frac{4}{n} \sum_{j=1}^{n-1} \mathrm{e}^{-\theta j}\left|E\left(X_{j}^{2}\right)-f_{H}(\theta)\right| \sum_{k=j+1}^{n} \mathrm{e}^{-\theta(k-j)}|\rho(k)| \\
& \quad+\frac{4\left|f_{H}(\theta)\right|}{n} \sum_{j=1}^{n-1} \mathrm{e}^{-\theta j} \sum_{k=j+1}^{n} \mathrm{e}^{-\theta(k-j)}|\rho(k)| \\
\leqslant & C n^{-1}
\end{aligned}
$$

For $E_{2}^{4}(n)$, since

$$
\frac{4}{n} \sum_{j=1}^{n-1} \sum_{k=j+1}^{n} \mathrm{e}^{-\theta(k-j)}|\rho(j)|\left|E\left(X_{j}^{2}\right)-f_{H}(\theta)\right| \mathrm{e}^{-\theta k} \leqslant \frac{C}{n} \sum_{j=1}^{n-1} j^{4 H-4} \leqslant \frac{C}{n},
$$

and

$$
\frac{4}{n} \sum_{j=1}^{n-1}|\rho(j)| \sum_{k=j+1}^{n} \mathrm{e}^{-\theta(k-j)} \mathrm{e}^{-\theta k} \leqslant \frac{C}{n} \sum_{j=1}^{n-1} j^{2 H-2} \leqslant C \varphi_{H}(n)
$$

we get

$$
\left|E_{2}^{4}(n)\right| \leqslant C \varphi_{H}(n)
$$

Thus, we conclude

$$
\left|E_{2}(n)-4 f_{H}(\theta)\left(\sum_{i=1}^{\infty} \mathrm{e}^{-\theta i} \rho(i)-\frac{f_{H}(\theta) e^{-2 \theta}}{1-\mathrm{e}^{-2 \theta}}\right)\right| \leqslant C \varphi_{H}(n) .
$$

For $E_{3}(n)$, since for every $\forall k>j,|D(k, j)| \leqslant C(j k)^{H-1}$, we can write

$$
\begin{aligned}
\left|E_{3}(n)\right| \leqslant & \frac{C}{n} \sum_{j=1}^{n-1} \sum_{k=j+1}^{n} \mathrm{e}^{-\theta(k-j)}\left|E\left(X_{j}^{2}\right)\right|(k j)^{H-1} \\
\leqslant & \frac{C}{n}\left[\sum_{j=1}^{n-1} \sum_{k=j+1}^{n} \mathrm{e}^{-\theta(k-j)}\left|E\left(X_{j}^{2}\right)-f_{H}(\theta)\right|(k j)^{H-1}\right. \\
& \left.+f_{H}(\theta) \sum_{j=1}^{n-1} \sum_{k=j+1}^{n} \mathrm{e}^{-\theta(k-j)}(k j)^{H-1}\right] \\
\leqslant & \frac{C}{n}\left[\sum_{j=1}^{n} j^{4 H-4}+\sum_{j=1}^{n-1} j^{2 H-2}\right] \\
\leqslant & C \varphi_{H}(n) .
\end{aligned}
$$

For $E_{4}(n)$, we first calculate $A(k, j)^{2}$. We have

$$
\begin{aligned}
A(k, j)^{2} & =\rho(k-j)^{2}-2 \rho(k-j) \mathrm{e}^{-\theta(k-j)} \rho(0)+\rho(0)^{2} \mathrm{e}^{-2 \theta(k-j)}+\mathrm{e}^{-2 \theta k} \rho(j)^{2} \\
& -2 \mathrm{e}^{-\theta(k+j)} \rho(j) \rho(k)+\mathrm{e}^{-2 \theta j} \rho(k)^{2}+2 \mathrm{e}^{-\theta k} \rho(k-j) \rho(j) \\
& -2 \mathrm{e}^{-\theta j} \rho(k) \rho(k-j)-2 \mathrm{e}^{-\theta(k-j)} \rho(0) \mathrm{e}^{-\theta k} \rho(j)+2 \mathrm{e}^{-\theta(k-j)} \rho(0) e^{-\theta j} \rho(k) .
\end{aligned}
$$


Hence, we need to study the rate of convergence of the following terms. We have

$$
\begin{aligned}
\left|\frac{2}{n} \sum_{j=1}^{n-1} \sum_{k=j+1}^{n} \rho(k-j)^{2}-2 \sum_{i=1}^{\infty} \rho(i)^{2}\right| & \leqslant 2 \sum_{i=n}^{\infty} i^{4 H-4}+\frac{2}{n} \sum_{i=1}^{n-1} i^{4 H-3} \\
\leqslant & C \psi_{H}(n), \\
4 \rho(0)\left|\frac{1}{n} \sum_{j=1}^{n-1} \sum_{k=j+1}^{n} \mathrm{e}^{-\theta(k-j)} \rho(k-j)-\sum_{i=1}^{\infty} \mathrm{e}^{-\theta i} \rho(i)\right| \leqslant & 4 \rho(0) \sum_{j=n}^{\infty} j^{2 H-2} \mathrm{e}^{-\theta j} \\
& +\frac{4 \rho(0)}{n} \sum_{i=1}^{n-1} \mathrm{e}^{-\theta i} i^{2 H-1} \\
\leqslant & C \varphi_{H}(n)
\end{aligned}
$$

and

$$
2 \rho(0)^{2}\left|\frac{1}{n} \sum_{j=1}^{n-1} \sum_{k=j+1}^{n} \mathrm{e}^{-2 \theta(k-j)}-\sum_{i=1}^{\infty} \mathrm{e}^{-2 \theta i}\right| \leqslant C n^{-1} .
$$

Moreover, it is clear that

$$
\frac{1}{n} \sum_{j=1}^{n-1} \sum_{k=j+1}^{n}\left[\mathrm{e}^{-2 \theta k} \rho(j)^{2}+\mathrm{e}^{-\theta(k+j)}|\rho(j)||\rho(k)|+\mathrm{e}^{-2 \theta j} \rho(k)^{2}\right] \leqslant C n^{-1}
$$

and

$$
\frac{1}{n} \sum_{j=1}^{n-1} \sum_{k=j+1}^{n} \mathrm{e}^{-\theta k}|\rho(k-j)||\rho(j)| \leqslant \frac{C}{n} \sum_{j=1}^{n-1} j^{2 H-2} \leqslant C \varphi_{H}(n) .
$$

In addition,

$$
\begin{aligned}
\frac{4}{n} \sum_{j=1}^{n-1} \sum_{k=j+1}^{n} \mathrm{e}^{-\theta j}|\rho(k)||\rho(k-j)| & \leqslant \frac{C}{n} \sum_{j=1}^{n-1} \mathrm{e}^{-\theta j} \sum_{k=j+1}^{n}(k-j)^{4 H-4} \\
& \leqslant \frac{C}{n} \sum_{i=1}^{\infty} i^{4 H-4} \sum_{j=1}^{n-1} \mathrm{e}^{-\theta j} \leqslant C n^{-1} \\
\frac{4}{n} \sum_{j=1}^{n-1} \sum_{k=j+1}^{n} \mathrm{e}^{-\theta(k-j)}|\rho(j)| \mathrm{e}^{-\theta k} & \leqslant \frac{4}{n} \sum_{j=1}^{n-1} j^{2 H-2} \sum_{k=j+1}^{n} \mathrm{e}^{-\theta(k-j)} \\
\frac{4 \rho(0)}{n} \sum_{j=1}^{n-1} \sum_{k=j+1}^{n} \mathrm{e}^{-\theta(k-j)}|\rho(k)| \mathrm{e}^{-\theta j} \leqslant & \frac{4 \rho(0)}{n} \sum_{j=1}^{n-1} \mathrm{e}^{-\theta j} \sum_{k=j+1}^{n} \mathrm{e}^{-\theta(k-j)}(k-j)^{2 H-2} \\
\leqslant & \frac{4 \rho(0)}{n} \sum_{j=1}^{n-1} e^{-\theta j} \sum_{i=1}^{\infty} \mathrm{e}^{-\theta i} \rho(i) \\
\leqslant & C n^{-1} .
\end{aligned}
$$


Thus,

$$
\left|E_{4}(n)-2 \sum_{i=1}^{\infty}\left(\rho(i)-f_{H}(\theta) \mathrm{e}^{-\theta i}\right)^{2}\right| \leqslant C \psi_{H}(n) .
$$

For $E_{5}(n)$, we have

$$
\begin{aligned}
\left|E_{5}(n)\right| & \leqslant \frac{C}{n} \sum_{j=1}^{n-1} \sum_{k=j+1}^{n}(k-j)^{2 H-2}(k j)^{H-1} \\
& \leqslant \frac{C}{n} \sum_{j=1}^{n-1} j^{H-1} \sum_{i=1}^{n-j} i^{2 H-2}(i+j)^{H-1} \\
& \leqslant \frac{C}{n}\left(\sum_{j=1}^{n} j^{2 H-2}\right)^{2} \leqslant C \psi_{H}(n) .
\end{aligned}
$$

Finally, using $D(k, j)^{2} \leqslant|A(k, j)||D(k, j)| \forall k>j$, we get

$$
\left|E_{6}(n)\right| \leqslant C\left|E_{5}(n)\right| \text {. }
$$

Combining (5.4), (5.5), (5.6), (5.7), (5.8) and (5.9) the proof is completed.

Let us now study the case when $H=3 / 4$.

Lemma 5.6. Let $X$ be the solution of (4.2). If $H=\frac{3}{4}$, we have

$$
\left|\frac{1}{n \log (n)} \sum_{\substack{j, k=1 \\ k \neq j}}^{n}\left(E\left[X_{k} X_{j}\right]\right)^{2}-\frac{9}{32 \theta^{4}}\right| \leqslant \frac{C}{\log (n)} .
$$

Proof: Using similar argument as in above, it is straightforward to check that

$$
\left|E_{1}(n)\right|+\left|E_{2}(n)\right|+\left|E_{3}(n)\right|+\left|E_{5}(n)\right|+\left|E_{6}(n)\right| \leqslant C,
$$

and

$$
\left|\frac{1}{\log (n)} E_{4}(n)-\frac{9}{32 \theta^{4}}\right| \leqslant C \log (n)^{-1}
$$

which completes the proof.

Lemma 5.7. Let $X$ be the solution of (4.1), with the process $G$ is a bifBm. If $0<H K<3 / 4$, we have

$$
\left|E\left[V_{n}(X)^{2}\right]-4 \sum_{i=1}^{\infty}\left[\rho(i)-\left(1-2^{1-K}\right) \mathrm{e}^{-\theta i} \rho(0)\right]^{2}-2^{3-2 K} \rho(0)^{2}\right| \leqslant C \psi_{H K}(n) .
$$

If $H K=3 / 4$, we have

$$
\left|\frac{E\left[V_{n}(X)^{2}\right]}{\log (n)}-\frac{9}{16 \theta^{4}}\right| \leqslant C \log (n)^{-1}
$$

Proof: We can write

$$
E\left[V_{n}(X)^{2}\right]=\frac{2}{n} \sum_{\substack{k, l=1 \\ k \neq l}}^{n}\left(E\left[X_{k} X_{j}\right]\right)^{2}+\frac{2}{n} \sum_{k=1}^{n}\left(E\left[X_{k}^{2}\right]\right)^{2}
$$


where

$$
\begin{aligned}
\left|\frac{1}{n} \sum_{k=1}^{n}\left(E\left[X_{k}^{2}\right]\right)^{2}-2^{2-2 K} \rho(0)^{2}\right| & \leqslant \frac{1}{n} \sum_{k=1}^{n}\left|E\left[X_{k}^{2}\right]-2^{1-K} \rho(0)\right|\left(E\left[X_{k}^{2}\right]+2^{1-K} \rho(0)\right) \\
& \leqslant \frac{C}{n} \sum_{k=1}^{n}\left|E\left[X_{k}^{2}\right]-2^{1-K} \rho(0)\right| \\
& \leqslant \frac{C}{n} \sum_{k=1}^{n} k^{2 H K-2} \\
& \leqslant C \varphi_{H K}(n) .
\end{aligned}
$$

Moreover,

$$
\left|\frac{1}{n} \sum_{\substack{j, k=1 \\ k \neq j}}^{n}\left(E\left[X_{k} X_{j}\right]\right)^{2}-2 \sum_{i=1}^{\infty}\left(\rho(i)-\left(1-2^{1-K}\right) \mathrm{e}^{-\theta i} \rho(0)\right)^{2}\right| \leqslant C \psi_{H K}(n) .
$$

Indeed, we have

$$
\frac{1}{n} \sum_{k, j=1}^{n}\left(E\left[X_{k} X_{j}\right]\right)^{2}=\bar{E}_{1}(n)+\bar{E}_{2}(n)+\bar{E}_{3}(n)+\bar{E}_{4}(n)+\bar{E}_{5}(n)+\bar{E}_{6}(n)
$$

where

$$
\begin{array}{ll}
\bar{E}_{1}(n):=\frac{2}{n} \sum_{j=1}^{n-1} \sum_{k=j+1}^{n} a(k, j)^{2}, \quad \bar{E}_{2}(n):=\frac{4}{n} \sum_{j=1}^{n-1} \sum_{k=j+1}^{n} a(k, j) \bar{A}(k, j) \\
\bar{E}_{3}(n):=\frac{4}{n} \sum_{j=1}^{n-1} \sum_{k=j+1}^{n} a(k, j) \bar{D}(k, j), \quad \bar{E}_{4}(n):=\frac{2}{n} \sum_{j=1}^{n-1} \sum_{k=j+1}^{n} \bar{A}(k, j)^{2} \\
\bar{E}_{5}(n):=\frac{4}{n} \sum_{j=1}^{n-1} \sum_{k=j+1}^{n} \bar{A}(k, j) \bar{D}(k, j), & \bar{E}_{6}(n):=\frac{2}{n} \sum_{j=1}^{n-1} \sum_{k=j+1}^{n} \bar{D}(k, j)^{2}
\end{array}
$$

with for $0<s<t$,

$$
\begin{aligned}
& a(s, t):=\mathrm{e}^{-\theta(t-s)} E\left[X_{s}^{2}\right], \\
& \bar{A}(t, s):=2^{1-K} H K(2 H K-1) \mathrm{e}^{-\theta t} \mathrm{e}^{-\theta s} \int_{s}^{t} \mathrm{e}^{\theta v} \int_{0}^{s} \mathrm{e}^{\theta u}(u-v)^{2 H K-2} d u d v, \\
& \bar{D}(t, s):=2^{2-K} K(K-1) \mathrm{e}^{-\theta t} \mathrm{e}^{-\theta s} \int_{s}^{t} \int_{0}^{s} \mathrm{e}^{\theta u} \mathrm{e}^{\theta v}(u v)^{2 H-1}\left(u^{2 H}+v^{2 H}\right)^{K-2} d u d v .
\end{aligned}
$$

Using similar arguments as in the subfractional case, we obtain

$$
\begin{aligned}
& || \bar{E}_{1}(n)-\frac{2^{3-2 K} \rho(0)^{2} \mathrm{e}^{-2 \theta}}{1-\mathrm{e}^{-2 \theta}} \mid \\
& +\left|\bar{E}_{2}(n)-2^{3-K} \rho(0) \sum_{i=1}^{\infty} \mathrm{e}^{-\theta i} \rho(i)-2^{3-K} \rho(0)^{2} \frac{\mathrm{e}^{-2 \theta}}{1-\mathrm{e}^{-2 \theta}}\right| \\
& +\left|\bar{E}_{3}(n)\right|+\left|\bar{E}_{4}(n)-2 \sum_{i=1}^{\infty}\left(\rho(i)-\rho(0) \mathrm{e}^{-\theta i}\right)^{2}\right|+\left|\bar{E}_{5}(n)\right|+\left|\bar{E}_{6}(n)\right| \\
& \leqslant C \psi_{H K}(n)
\end{aligned}
$$


which completes the proof of the first inequality of Lemma 5.7.

By using similar techniques as in above we also obtain for $H K=3 / 4$,

$$
\left|\frac{1}{n \log (n)} \sum_{\substack{j, k=1 \\ k \neq j}}^{n}\left(E\left[X_{k} X_{j}\right]\right)^{2}-\frac{9}{32} \frac{1}{\theta^{4}}\right| \leqslant C \log (n)^{-1}
$$

which finishes the proof.

\section{Acknowledgments}

The authors would like to thank the anonymous referee for his/her careful reading and valuable suggestions that improved the results of the paper.

\section{References}

E. Azmoodeh and J. I. Morlanes. Drift parameter estimation for fractional OrnsteinUhlenbeck process of the second kind. Statistics 49 (1), 1-18 (2015). MR3304364.

E. Azmoodeh and L. Viitasaari. Parameter estimation based on discrete observations of fractional Ornstein-Uhlenbeck process of the second kind. Stat. Inference Stoch. Process. 18 (3), 205-227 (2015). MR3395605.

R. Belfadli, K. Es-Sebaiy and Y. Ouknine. Parameter estimation for fractional Ornstein-Uhlenbeck processes: Non-ergodic case. Frontiers in Science and Engineering (An International Journal Edited by Hassan II Academy of Science and Technology) 1 (1), 1-16 (2011).

H. Biermé, A. Bonami, I. Nourdin and G. Peccati. Optimal Berry-Esseen rates on the Wiener space: the barrier of third and fourth cumulants. ALEA Lat. Am. J. Probab. Math. Stat. 9 (2), 473-500 (2012). MR3069374.

A. Brouste and S. M. Iacus. Parameter estimation for the discretely observed fractional Ornstein-Uhlenbeck process and the Yuima R package. Comput. Statist. 28 (4), 1529-1547 (2013). MR3120827.

P. Cheridito, H. Kawaguchi and M. Maejima. Fractional Ornstein-Uhlenbeck processes. Electron. J. Probab. 8, no. 3, 14 (2003). MR1961165.

M. El Machkouri, K. Es-Sebaiy and Y. Ouknine. Least squares estimator for nonergodic Ornstein-Uhlenbeck processes driven by Gaussian processes. J. Korean Statist. Soc. 45 (3), 329-341 (2016). MR3527650.

B. El Onsy, K. Es-Sebaiy and C. A. Tudor. Statistical analysis of the non-ergodic fractional Ornstein-Uhlenbeck process of the second kind. Commun. Stoch. Anal. 11 (2), Article 1, 119-136 (2017a). MR3687770.

B. El Onsy, K. Es-Sebaiy and F. G. Viens. Parameter estimation for a partially observed Ornstein-Uhlenbeck process with long-memory noise. Stochastics 89 (2), 431-468 (2017b). MR3590429.

K. Es-Sebaiy and F. Viens. Optimal rates for parameter estimation of stationary Gaussian processes (2019+). To appear in Stoc. Process. Appl.

$\mathrm{X}$. Fernique. Regularité des trajectoires des fonctions aléatoires gaussiennes pages 1-96. Lecture Notes in Math., Vol. 480 (1975). MR0413238.

Y. Hu and D. Nualart. Parameter estimation for fractional Ornstein-Uhlenbeck processes. Statist. Probab. Lett. 80 (11-12), 1030-1038 (2010). MR2638974. 
Y. Hu and J. Song. Parameter estimation for fractional Ornstein-Uhlenbeck processes with discrete observations. In Malliavin calculus and stochastic analysis, volume 34 of Springer Proc. Math. Stat., pages 427-442. Springer, New York (2013). MR3070455.

P. E. Kloeden and A. Neuenkirch. The pathwise convergence of approximation schemes for stochastic differential equations. LMS J. Comput. Math. 10, 235253 (2007). MR2320830.

A. Neuenkirch and S. Tindel. A least square-type procedure for parameter estimation in stochastic differential equations with additive fractional noise. Stat. Inference Stoch. Process. 17 (1), 99-120 (2014). MR3178015.

L. Neufcourt and F. G. Viens. A third-moment theorem and precise asymptotics for variations of stationary Gaussian sequences. ALEA Lat. Am. J. Probab. Math. Stat. 13 (1), 239-264 (2016). MR3479476.

I. Nourdin and G. Peccati. Normal approximations with Malliavin calculus, volume 192 of Cambridge Tracts in Mathematics. Cambridge University Press, Cambridge (2012). ISBN 978-1-107-01777-1. From Stein's method to universality.

I. Nourdin and G. Peccati. The optimal fourth moment theorem. Proc. Amer. Math. Soc. 143 (7), 3123-3133 (2015). MR3336636.

D. Nualart. The Malliavin calculus and related topics. Probability and its Applications (New York). Springer-Verlag, Berlin, second edition (2006). ISBN 978-3-540-28328-7; 3-540-28328-5. MR2200233.

D. Nualart and G. Peccati. Central limit theorems for sequences of multiple stochastic integrals. Ann. Probab. 33 (1), 177-193 (2005). MR2118863.

D. Nualart and H. Zhou. Total variation estimates in the Breuer-Major theorem. ArXiv Mathematics e-prints (2018). arXiv: 1807.09707.

F. G. Viens and A. B. Vizcarra. Supremum concentration inequality and modulus of continuity for sub- $n$th chaos processes. J. Funct. Anal. 248 (1), 1-26 (2007). MR2329681.

L. C. Young. An inequality of the Hölder type, connected with Stieltjes integration. Acta Math. 67 (1), 251-282 (1936). MR1555421. 